\title{
Live cell screening platform identifies PPAR $\delta$ as a regulator of cardiomyocyte proliferation and cardiac repair
}

Ajit Magadum ${ }^{1,2,{ }^{*}}$, Yishu Ding ${ }^{3,}$, Lan $\mathrm{He}^{3}$, Teayoun Kim ${ }^{3}$, Mohankrishna Dalvoy Vasudevarao ${ }^{4}$, Qinqiang Long, Kevin Yang ${ }^{3}$, Nadeera Wickramasinghe ${ }^{6}$, Harsha V Renikunta ${ }^{1}$, Nicole Dubois ${ }^{6}$, Gilbert Weidinger ${ }^{4}$, Qinglin Yang ${ }^{3,5}$, Felix B Engel ${ }^{1,7,8}$

${ }^{I}$ Department of Cardiac Development and Remodelling, Max-Planck-Institute for Heart and Lung Research, Parkstrasse 1, Bad Nauheim 61231, Germany; ${ }^{2}$ Department of Cardiology, Icahn School of Medicine at Mount Sinai Hospital, One Gustave L. Levy Place, Box 1030, New York, NY 10029, USA; ${ }^{3}$ Department of Nutrition Sciences, University of Alabama at Birmingham, 1675 University Blvd, Birmingham, AL 35294-3360, USA; ${ }^{4}$ Institute of Biochemistry and Molecular Biology, Ulm University, Albert-Einstein-Allee 11, Ulm 89081, Germany; ${ }^{5}$ Division of Cardiology, Department of Internal Medicine, Tongji Hospital, Tongji Medical College, Huazhong University of Science and Technology, 1095 Jiefang Ave, Wuhan, Hubei 430030, China; ${ }^{6}$ Department for Cell, Developmental and Regenerative Biology, Icahn School of Medicine at Mount Sinai, 1470 Madison Avenue, Box 1040, New York, NY 10029, USA; 'Department of Nephropathology, Experimental Renal and Cardiovascular Research, Institute of Pathology, Friedrich-Alexander-Universität Erlangen-Nürnberg, Schwabachanlage 12, Erlangen 91054, Germany; ${ }^{8}$ Muscle Research Center Erlangen (MURCE)

Zebrafish can efficiently regenerate their heart through cardiomyocyte proliferation. In contrast, mammalian cardiomyocytes stop proliferating shortly after birth, limiting the regenerative capacity of the postnatal mammalian heart. Therefore, if the endogenous potential of postnatal cardiomyocyte proliferation could be enhanced, it could offer a promising future therapy for heart failure patients. Here, we set out to systematically identify small molecules triggering postnatal cardiomyocyte proliferation. By screening chemical compound libraries utilizing a Fucci-based system for assessing cell cycle stages, we identified carbacyclin as an inducer of postnatal cardiomyocyte proliferation. In vitro, carbacyclin induced proliferation of neonatal and adult mononuclear rat cardiomyocytes via a per-

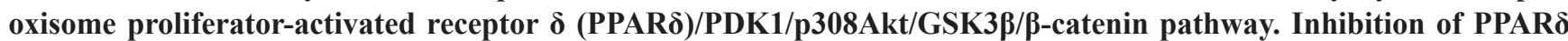
reduced cardiomyocyte proliferation during zebrafish heart regeneration. Notably, inducible cardiomyocyte-specific overexpression of constitutively active PPAR $\delta$ as well as treatment with PPAR $\delta$ agonist after myocardial infarction in mice induced cell cycle progression in cardiomyocytes, reduced scarring, and improved cardiac function. Collectively, we established a cardiomyocyte proliferation screening system and present a new drugable target with promise for the treatment of cardiac pathologies caused by cardiomyocyte loss.

Keywords: cardiac repair; cardiomyocyte proliferation; screening; carbacyclin; PPAR $\delta$; GSK3 $\beta$; Tbx20

Cell Research (2017) 27:1002-1019. doi:10.1038/cr.2017.84; published online 16 June 2017

\section{Introduction}

It has been projected that the future socio-economic burden of heart disease will further increase due to aging

*These two authors contributed equally to this work.

Correspondence: Felix B Engel ${ }^{\mathrm{a}}$, Qinglin Yang ${ }^{\mathrm{b}}$

${ }^{a}$ Tel: +49-0-9131-85 25699

E-mail: felix.engel@uk-erlangen.de

bE-mail: qyang@uab.edu

Received 11 October 2016; revised 5 May 2017; accepted 8 May 2017; published online 16 June 2017 populations in high-income countries and to the increasing incidence of cardiac risk factors such as hypertension, obesity and diabetes in low-income countries. Thus, it is important to identify strategies for repairing the heart because conventional treatment regimens fail to prevent cardiomyocyte loss and cannot reverse cardiac damage [1].

Zebrafish can regenerate their adult heart through cardiomyocyte proliferation [2]. Yet, in the mammalian heart, regeneration is limited to newborns because cardiomyocyte proliferation rapidly ceases after birth [3]. 
Why mammalian cardiomyocytes stop proliferating after birth and whether this block is permanent is unclear. Recently, several explanations have been put forward including gene silencing [4], DNA damage [5] and centrosome disassembly [6]. While these data suggest that the cell cycle arrest in postnatal cardiomyocytes is permanent, several publications have provided evidence that induction of cardiomyocyte proliferation in the adult mammalian heart improves cardiac function [7-9]. Thus, there might be a subpopulation of mammalian cardiomyocytes, as previously suggested $[10,11]$, that behaves like adult zebrafish cardiomyocytes. Importantly, therapy for heart failure is elusive and even in zebrafish, the mechanism of cardiac regeneration is still poorly understood.

Here, we set out to systematically identify small molecules triggering proliferation of neonatal mononuclear rat cardiomyocytes on the assumption that signaling pathways required for proliferation of zebrafish cardiomyocytes are conserved and would act to promote proliferation of a presumptive mononuclear adult cardiomyocyte subpopulation in mammals. For this purpose we established a simple, fast, affordable and efficient cardiomyocyte-specific screening system based on the Fucci system in which cells at specific phases of the cell cycle express phase-specific fluorescent markers [12]. Our screen and subsequent animal experiments identified the peroxisome proliferator-activated receptor delta (PPAR $\delta$, also known as PPAR $\beta$ ), a nuclear hormone receptor and transcription factor [13], as a promising target for therapeutic induction of cardiomyocyte proliferation. PPAR $\delta$ has previously been associated with a variety of proliferative diseases that include hyperproliferative skin disorders and cancer as well as with proliferation and cell survival of several cell types [14, $15]$. PPAR $\delta$ has also been linked to wound healing and tissue repair due to cell proliferation in mammalian skin, liver, muscle and cornea [16-19]. The roles of PPAR $\delta$ in transcriptional control of physiological and pathological processes have been recently reviewed [13].

PPAR $\delta$ is highly expressed in the embryonic as well as adult heart and known as a powerful regulator of fatty acid catabolism and energy homeostasis [20, 21]. PPAR $\delta$ knockout leads to embryonic lethality and growth retardation in the surviving mice $[22,23]$. The role of PPAR $\delta$ has previously also been investigated in different heart diseases [24]. For instance, we have shown that cardiomyocyte-restricted PPAR $\delta$ deletion in mice perturbs myocardial fatty acid oxidation causing cardiac dysfunction leading to hypertrophy, fibrosis and lipotoxic cardiomyopathy [25]. In contrast, cardiac-specific overexpression of PPAR $\delta$ is protective to ischemia/reperfusion (I/R) injury as well as TAC-induced pressure overload [26, 27]. However, there are no reports on the role of PPAR $\delta$ in cardiomyocyte pro- liferation in vitro or in vivo. Here we show that carbacyclin induces cardiomyocyte proliferation via a PPAR $\delta / P D K 1 /$ p308Akt/GSK3 $\beta / \beta$-catenin-pathway and that activated PPAR $\delta$ signaling after myocardial infarction (MI) induces cardiomyocyte cell cycle activity and improves scarring as well as cardiac function. Moreover, inhibition of PPAR $\delta$ reduces cardiomyocyte proliferation during zebrafish heart regeneration.

\section{Results}

Screening for small molecules promoting cardiomyocyte proliferation in vitro

The so-called Fucci system, developed to monitor cell cycle progression, makes use of the fact that Cdt1 (a marker of G1/G0 phase) and Geminin (a marker of S, G2 and M phases) are subject to cell cycle-dependent proteolysis [12]. Thus, the Fucci system can be used to screen for potential inducers of cell cycle re-entry through either loss of the Cdt1 signal or gain of the Geminin signal (Supplementary information, Figure S1A).

We first considered monitoring the loss of Cdt1 signal. Adenovirus (Ad)-mediated transfection of primary postnatal Day 3 (P3) rat cardiomyocytes with a non-functional hCdt1 deletion mutant fused to mCherry (mCherry-hC$\mathrm{dt} 1(30 / 120))$ under the control of the cardiomyocyte-specific $\alpha$-MHC promoter (Ad-mCherry-hCdt1(30/120) resulted in the expression of mCherry-hCdt1(30/120) in $>90 \%$ of neonatal cardiomyocytes based on mCherry staining and counterstaining against cardiomyocyte-specific Troponin I (Supplementary information, Figure S1B-S1D). However, a markedly lower number of mCherry-hCdt1(30/120)-positive cardiomyocytes were detected in observations of live cells (Supplementary information, Figure S1E). In addition, screening based on the decrease in the number of mCherry-hCdt1(30/120)-positive cells appears to be error prone due to the unavoidable presence of non-myocytes; drug-induced non-myocyte proliferation will result in a "false loss of signal" (Supplementary information, Figure S1F). Thus, we did not further pursue loss of Cdt1 as a readout for induction of cardiomyocyte proliferation.

We then turned to monitoring Geminin expression. Immunofluorescence analyses revealed that adenoviral overexpression of a non-functional human Geminin deletion mutant fused to a monomeric version of Azami Green (mAG-hGem(1/110)) in P3 rat cardiomyocytes in vitro under the control of the $\alpha$-MHC promoter resulted in the expression of $\mathrm{mAG}-\mathrm{hGem}(1 / 110)$ in $<1.5 \%$ of cardiomyocytes (Figure 1A and 1B). This suggests that overexpressed $\mathrm{mAG}-\mathrm{hGem}(1 / 110)$ is actively degraded in P3 cardiomyocytes arrested in G1/G0 phase. Stimulation with FGF1 and the p38 inhibitor SB203580 (p38i), which 

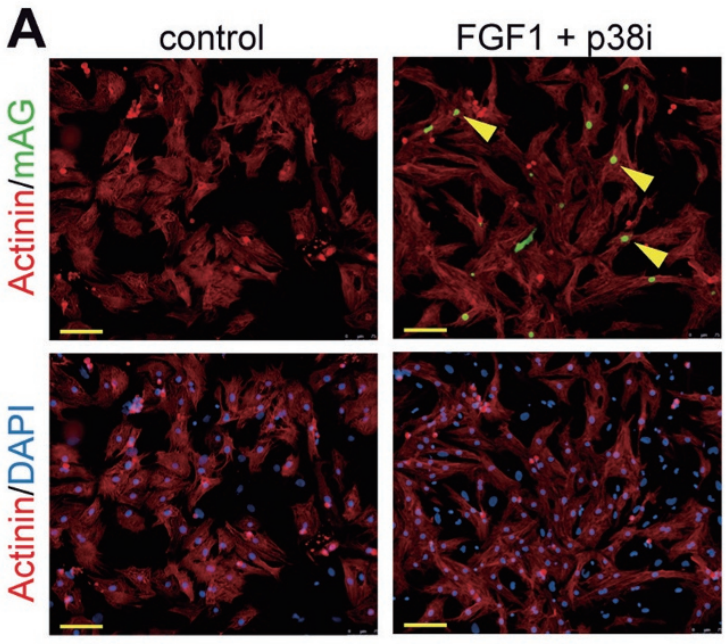

E

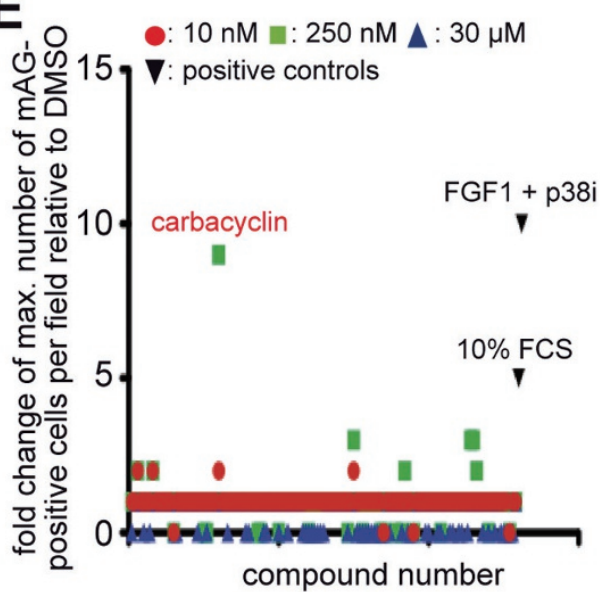

B

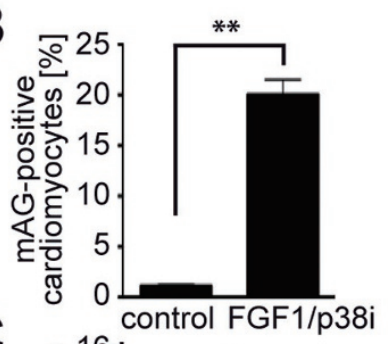

C

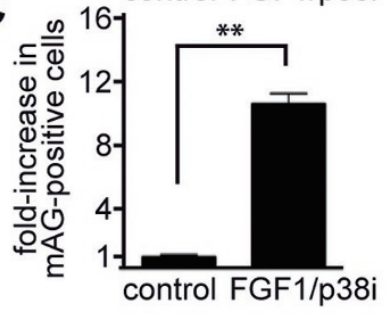

$\mathbf{F}$
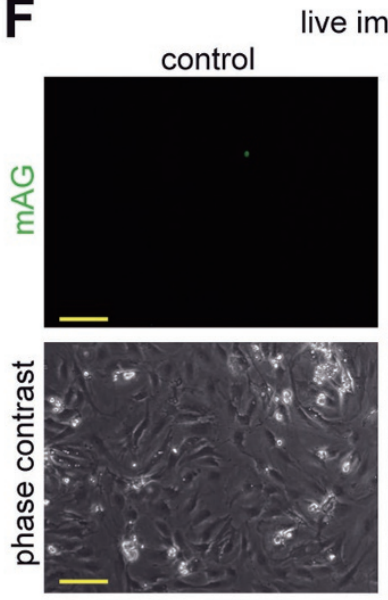

D

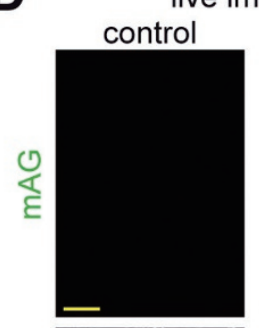

live imaging
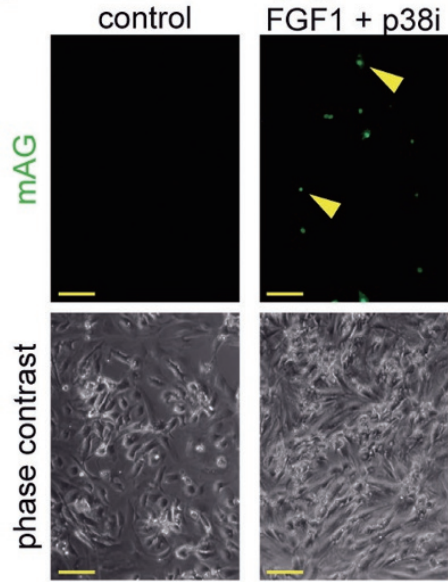

G

Figure 1 Chemical library screen identifies carbacyclin as a potential inducer of cardiomyocyte proliferation. (A) Representative examples of Ad-mAG-hGem(1/110) infected postnatal cardiomyocyte cultures after serum starvation (control) or stimulation with FGF1/p38i stained for mAG expression (green), cardiomyocyte-specific Actinin (red), and DNA (DAPI, blue). Yellow arrowheads, mAG-positive cardiomyocyte. (B) Quantitative analysis of $\mathbf{A}(n=6)$. (C, D) Quantitative analysis $(n=6)$ and representative live images of control or FGF1 + p38i-stimulated cardiomyocyte cultures infected with Ad-mAG-hGem(1/110) (green). (E) Quantitative analysis of the screen of a nuclear receptor ligand library and an epigenetics screening library. (F, G) Quantitative analysis $(n=6)$ and representative live images of control- or carbacyclin-treated neonatal cardiomyocyte cultures infected with Ad-mAG-hGem(1/110) (green). Yellow arrowheads: mAG-positive cells. $* * P<0.01$. Scale bar $=100 \mu \mathrm{m}$.

have been shown to efficiently induce $\mathrm{P} 3$ rat cardiomyocyte proliferation [28], increased mAG-hGem(1/110) expression in cardiomyocytes transfected with Ad-mAGhGem(1/110) by approximately 18 -fold compared to the control (Figure 1A and 1B). FGF1/p38i-induced mAGhGem(1/110) expression could also be easily detected by visual inspection without the need of immunofluorescence analysis. The number of $\mathrm{mAG}-\mathrm{hGem}(1 / 110)$-positive cells per microscopic field was increased by approximately 10 fold compared to the control (Figure 1C and 1D). This suggests that Geminin induction would provide a better live imaging screening system to identify small molecules with the potential to promote cardiomyocyte proliferation than loss of Cdt1.

We therefore chose to use this approach to screen a nuclear receptor ligand library (74 compounds) and an epigenetics screening library (54 compounds) in a 96well plate format at three different concentrations (Figure $1 \mathrm{E}$ ) in the presence of $0.2 \%$ fetal calf serum (FCS). To this end, we infected $\mathrm{P} 3$ rat cardiomyocytes with AdmAG-hGem(1/110) (infection efficiency $>90 \%$ ) using DMSO treatment as negative control. To induce cell cycle activity as a positive control, we treated cells with either $10 \%$ FCS, which induced a 5 -fold increase in mAG-hGem (1/110)-positive cells per field, or FGF1/p38i, which induced an approximate 10-fold increase in cells expressing 
this marker. We found that 8 compounds induced at least a 2-fold increase in mAG-hGem-positive cells (Figure $1 \mathrm{E}$ and Supplementary information, Table S1). The most potent treatment was $250 \mathrm{nM}$ carbacyclin, which induced an approximate 9-fold increase (Figure 1E-1G). These data suggest that carbacyclin, a known potent agonist of $\operatorname{PPAR} \delta$, is a previously unknown inducer of mammalian postnatal cardiomyocyte proliferation.

To determine the optimal concentrations of the 8 identified compounds that would promote progression into $\mathrm{S}$ phase, we performed BrdU incorporation assays (Figure 2A, 2B and Supplementary information, Figure S2A$\mathrm{S} 2 \mathrm{G})$. Carbacyclin was the most potent compound tested and induced BrdU incorporation in a dose-dependent manner with an optimal concentration of $1 \mu \mathrm{M}(46.3 \% \pm 3.8 \%$ vs DMSO: $3.6 \% \pm 0.6 \%, P<0.01$, Figure $2 \mathrm{~A}$ and $2 \mathrm{~B}$ ). Furthermore, mAG-hGem(1/110)-positive P3 cardiomyocytes that had been infected with Ad-mAG-hGem(1/110) and treated with carbacyclin progressed into cytokinesis (Supplementary information, Figure S3A-S3C). Furthermore, carbacyclin induced the expression of positive regulators of cell cycle progression including phospho-RB, cyclin D2, cyclin A, cyclin B, cdc2 and c-myc, and downregulated the cell cycle inhibitors p21 and p27 (Figure $2 \mathrm{C}-2 \mathrm{~F})$. In addition, carbacyclin stimulation increased the number of cardiomyocytes positive for the mitosis/cytokinesis markers phophorylated histone $\mathrm{H} 3$ (H3P) and Aurora B by approximately 11 -fold within three days (Figure 2G-2I). We observed cardiomyocytes in all stages of the cycle, including the act of division through the breaking of the midbody resulting in two daughter cells. Moreover, the stimulated cells exhibited transient dedifferentiation of the sarcomeric apparatus during mitosis (Supplementary information, Figure S4A). Finally, although carbacyclin treatment did not induce cardiomyocyte binucleation (Figure 2J), it did result in a 2-fold increase in cardiomyocyte cell number within 7 days of culture (Figure 2K). Together, these data demonstrate that carbacyclin induces P3 rat cardiomyocyte proliferation. Notably, carbacyclin had no effect on cell cycle progression of non-myocytes in a non-enriched cardiomyocyte culture (Supplementary information, Figure S4B and S4C).

\section{Carbacyclin induces cardiomyocyte proliferation in vitro via PPAR}

Carbacyclin is a chemically stable carbocyclic ana$\log$ of prostacyclin, a known potent agonist of PPAR $\delta$. Indeed, treatment with GW0742, another agonist of $\operatorname{PPAR} \delta$, also substantially increased the number of mAG-hGem(1/110)-positive (Supplementary information, Figure S3D) and BrdU-positive cardiomyocytes (Figure 3A). Moreover, carbacyclin-induced BrdU incorporation
(Figure 3B) and mAG-hGem(1/110) expression (Supplementary information, Figure S3E) was markedly reduced by the PPAR $\delta$ inhibitor GSK3787. In addition, BrdU incorporation could be inhibited by siRNA-mediated knockdown of PPAR $\delta$ (Figure 3B). These data demonstrate that carbacyclin-mediated cardiomyocyte proliferation requires activation of PPAR $\delta$.

It has previously been demonstrated that the PPAR $\delta$ agonist GW501516 upregulates 3-phosphoinositide-dependent protein kinase-1 (PDK1) expression in vivo [29]. Thus, we assessed whether carbacyclin activates PPAR $\delta$ via PDK1 and phosphorylation of Akt in cardiomyocytes. Carbacyclin stimulation resulted in phosphorylation of Akt at Thr308 as well as upregulation of PPAR $\delta$ and PDK1 (Figure 3C and 3D). To determine if this pathway is required for carbacyclin-induced cardiomyocyte proliferation, we overexpressed dominant negative (DN)-Akt, which markedly decreased carbacyclin-induced BrdU incorporation (Figure 3E). Moreover, BrdU incorporation was also decreased after addition of $20 \mu \mathrm{M}$ PHT427, a dual inhibitor of PDK1/Akt. In contrast, neither $20 \mu \mathrm{M}$ ERK inhibitor PD98059 nor $10 \mu \mathrm{M}$ PI3 kinase inhibitor LY294002 affected carbacyclin-mediated BrdU incorporation (Figure 3E). In addition, carbacyclin increased BrdU incorporation in human-induced pluripotent stem cell (hiPSC)-derived cardiomyocytes (Supplementary information, Figure S5A and S5B). The addition of $20 \mu \mathrm{M}$ PHT427 markedly reduced carbacyclin-induced BrdU incorporation in hiPSC-derived cardiomyocytes (Supplementary information, Figure S5B). It also reduced mAGhGem(1/110) expression in Ad-mAG-hGem(1/110)infected P3 neonatal cardiomyocytes stimulated with carbacyclin (Supplementary information, Figure S3E). Finally, carbacyclin promoted mitosis and cytokinesis in hiPSC-derived cardiomyocytes (Supplementary information, Figure S5C-S5F). Taken together, these data indicate that carbacyclin promotes cardiomyocyte proliferation via a PPARס/PDK1/p308Akt pathway.

\section{PPAR inhibition reduces cardiomyocyte proliferation during zebrafish heart regeneration \\ Our strategy to find new compounds to induce mam- malian heart repair is based on the hypothesis that pro- liferation signaling pathways are conserved in zebrafish cardiomyocytes, $\mathrm{P} 3$ rat cardiomyocytes and a presumptive mononuclear adult mammalian cardiomyocyte subpopula- tion. If correct, this would infer that PPAR $\delta$ plays a role in zebrafish heart regeneration. To test this, we subjected ze- brafish heart to cryoinjury and 6 days later (6 dpi) inhibit- ed PPAR $\delta$ activity by a $24 \mathrm{~h}$ treatment with GSK3787 be- fore assessing cardiomyocyte proliferation by proliferat- ing cell nuclear antigen (PCNA) expression. The fraction}



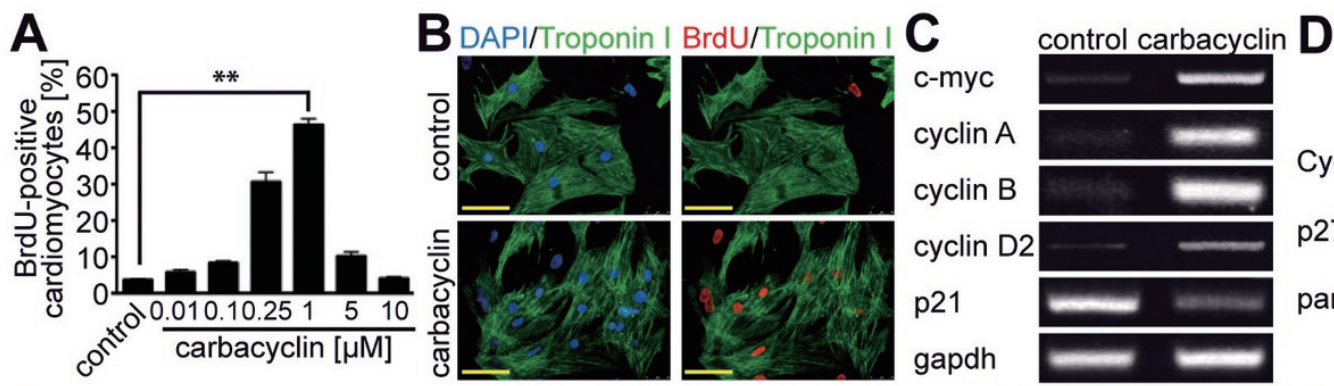

E
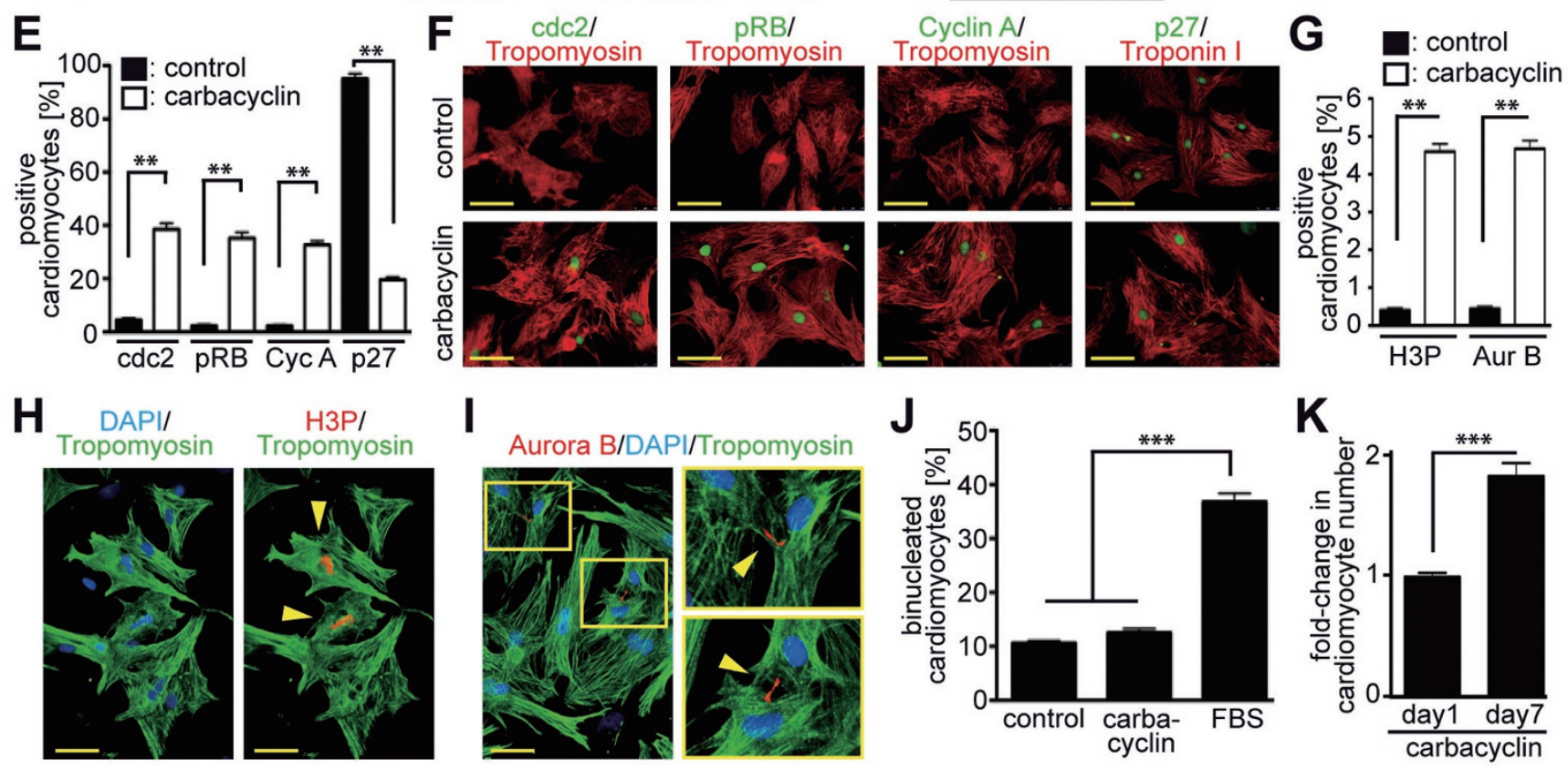

Figure 2 Validation of carbacyclin as an inducer of neonatal cardiomyocyte proliferation. (A, B) Representative immunofluorescence images and quantitative analysis $(n=6)$ showing that carbacyclin induces dose-dependent BrdU incorporation (red) into cardiomyocytes (Troponin I, green). (C-F) Treatment with carbacyclin induces increased expression of cell cycle promoting factors and decreased expression of cell cycle inhibitors (C: RT-PCR; D: western blot; E and F: immunofluorescence analyses at $48 \mathrm{~h}$ after stimulation with quantitative analysis, $n=6)$. (G-I) Carbacyclin treatment induces mitosis and cytokinesis in neonatal cardiomyocytes. (G) Quantitative analysis of H3P- and Aurora B (Aur B)-positive cardiomyocytes after carbacyclin stimulation $(1 \mu \mathrm{M})(n=6)$. $(\mathbf{H}, \mathrm{I})$ Representative examples of neonatal cardiomyocytes in mitosis (H3P-positive, red) or cytokinesis (Aurora B-positive, red) (Troponin I, green). DNA was visualized using DAPI (blue). (J) Quantitative analysis of binucleation after carbacyclin stimulation after 5 days of treatment (cells were treated on Day 0 and Day 3$)(n=4)$. (K) Quantitative analysis of cell count experiments $(n=6)$. ${ }^{* *} P<0.01,{ }^{* * *} P<0.001$. Scale bar $=50 \mu \mathrm{m}$.

of PCNA-positive cardiomyocytes at the wound border was significantly reduced upon treatment with PPAR $\delta$ antagonist (Figure 4A and 4B). In addition, inhibition of PPAR $\delta$ activity led to a reduction in cardiomyocyte mitosis as determined by H3P staining (Supplementary information, Figure S6). We conclude that endogenous $\operatorname{PPAR} \delta$ activity is required for naturally occurring regenerative cardiomyocyte proliferation in zebrafish.

Activation of PPARS induces adult cardiomyocyte cell cycle re-entry in vitro

To determine if PPAR $\delta$ activation can also promote cell cycle progression in more differentiated mammalian cardiomyocytes, we stimulated cardiomyocytes of older animals with carbacyclin. This treatment increased BrdU incorporation in $\mathrm{P} 8$ cardiomyocytes in vitro by more than 5 -fold $(14.5 \% \pm 1.7 \%$ vs $2.73 \% \pm 0.4 \%, P<0.01$; Figure 5A), with mainly mononucleated cardiomyocytes incorporating BrdU (Figure 5B). The mitotic index (H3P-positive) was increased by almost 7 -fold $(1.16 \%$ $\pm 0.13 \%$ vs $0.17 \% \pm 0.04 \%, P<0.01$; Figure 5 C). Carbacyclin treatment of cardiomyocytes from 12-week-old rats increased $\mathrm{mAG}-\mathrm{hGem}(1 / 110)$ expression at Day 5 of culture by greater than 150 -fold from $0.01 \%$ (follow- 

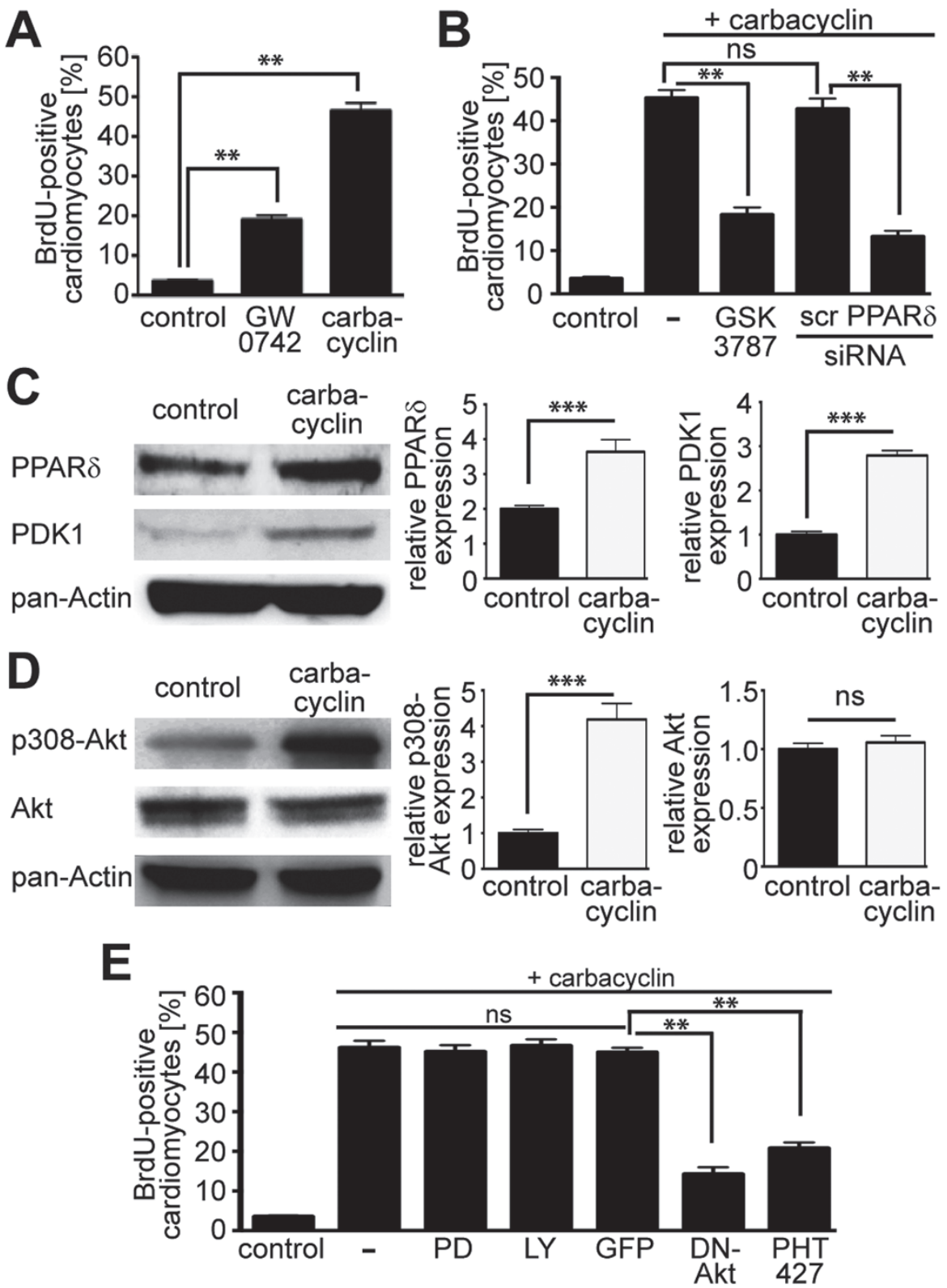

Figure 3 Carbacyclin induces cardiomyocyte proliferation via PPAR $\delta$. (A) Two agonists of PPAR $\delta$, carbacyclin and GW0742 significantly increase DNA synthesis in neonatal cardiomyocytes. (B) Inhibition of PPAR $\delta$ by siRNA or the antagonist GSK3787 markedly reduces the effect of carbacyclin on cardiomyocyte DNA synthesis (scr: scrambled). (C, D) Representative examples of western blot analysis including densitometric quantification $(n=3)$ showing that carbacyclin increases the expression of PPAR $\delta$ at $48 \mathrm{~h}$ and PDK1 at $24 \mathrm{~h}$ as well as (C) phosphorylation of Akt (p308Akt) after 1h (D). (E) Akt phosphorylation at position 308 is required for carbacyclin-induced DNA synthesis (dominant negative (DN)-Akt, PHT427) but not PI3 kinase-induced Akt phosphorylation (LY 294002) or ERK activity (PD 98059). ${ }^{* *} P<0.01,{ }^{* * *} P<0.001$.

ing DMSO treatment) to $1.5 \% \pm 0.2 \%$ (Figure $5 \mathrm{D}$ ). This resulted in $1.4 \% \pm 0.2 \%$ BrdU-positive cardiomyocytes at Day 6 (labeled for the final 5 days of culture) (Figure $5 \mathrm{D}$ and $5 \mathrm{E})$. To assess cell division, we analyzed 100 000 adult cardiomyocytes each from 4 independent experiments and found in the control an average of one
H3P-positive and $(1 ; 0 ; 2 ; 1)$ and one Aurora B-positive $(2 ; 2 ; 0 ; 0)$ cell. In contrast, after carbacyclin stimulation we detected an average of $58 \mathrm{H3P}$-positive $(64 ; 55 ; 60$; $54)$ and 34 Aurora B-positive $(31 ; 34 ; 39 ; 32)$ adult cardiomyocytes (Figure 5F and $5 \mathrm{G}$ ). Notably, $58.6 \% \pm 5.0 \%$ of the BrdU-positive, $58.1 \% \pm 6.4 \%$ of the H3P-positive 

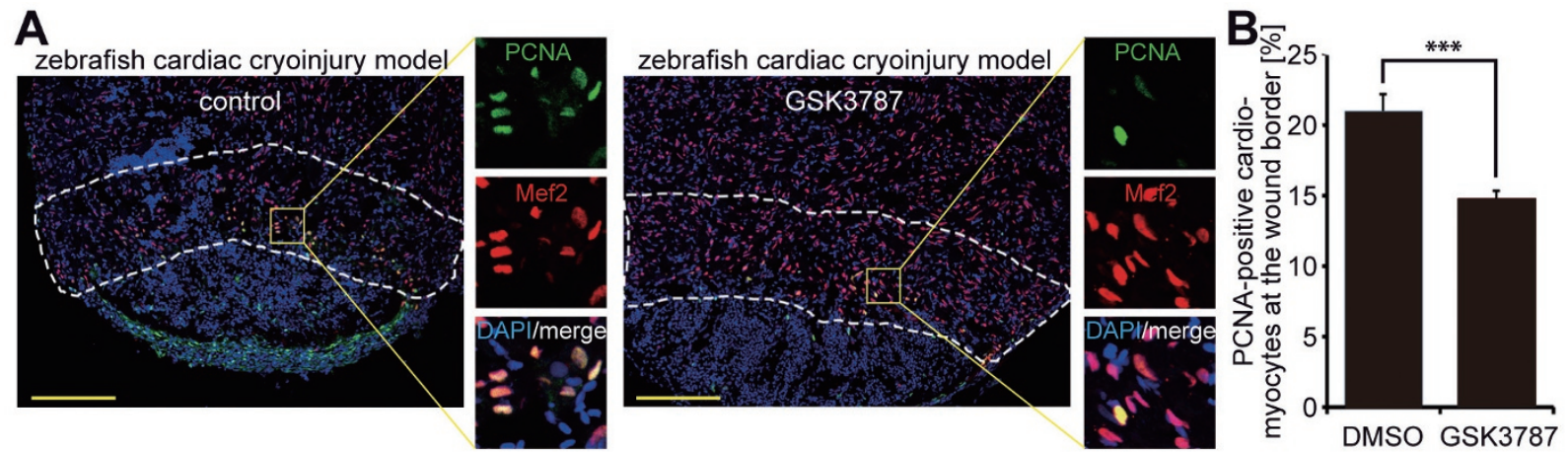

Figure 4 PPAR $\delta$ activity is required for adult cardiomyocyte cell cycle activity after injury in zebrafish. (A) Representative images of cryoinjured zebrafish hearts (7 dpi) treated with DMSO (control) or $5 \mu \mathrm{M}$ PPAR $\delta$ inhibitor (GSK3787), stained for PCNA (green) and the cardiomyocyte marker Mef2 (red). Nuclei were visualized using DAPI (blue). Dashed lines highlight the wound border zone used for quantification. Scale bar $=150 \mu \mathrm{m}$. (B) Quantitative analysis of PCNA-positive cardiomyocytes (DMSO: $n=9$ hearts; GSK3787: $n=10$ hearts; $* * * P<0.001$ ).
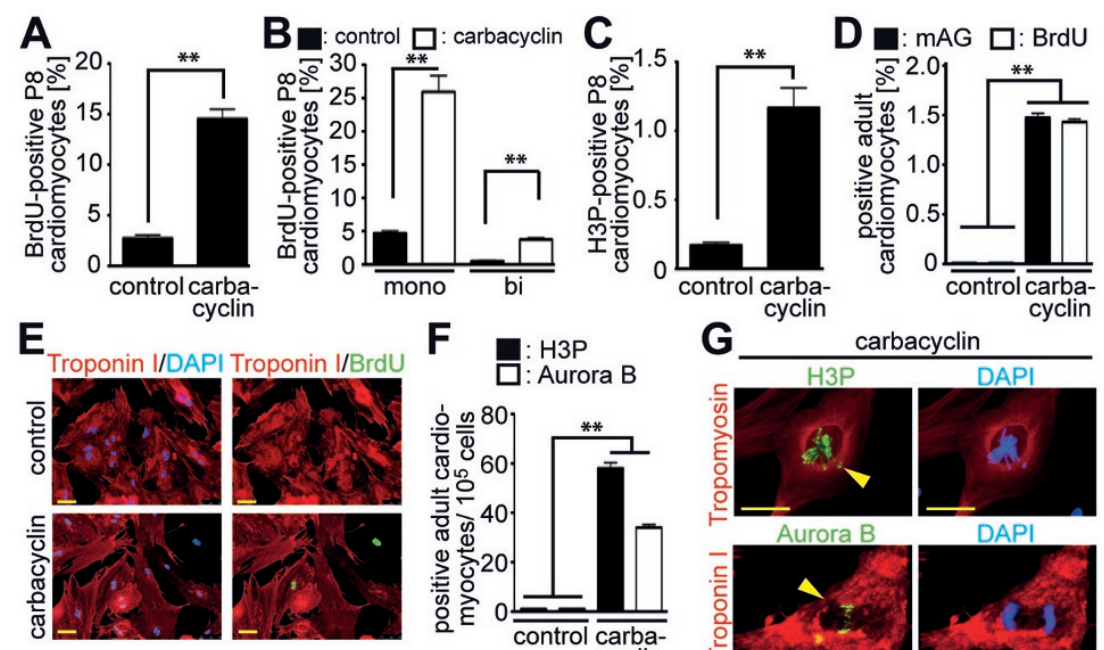

carbacyclin
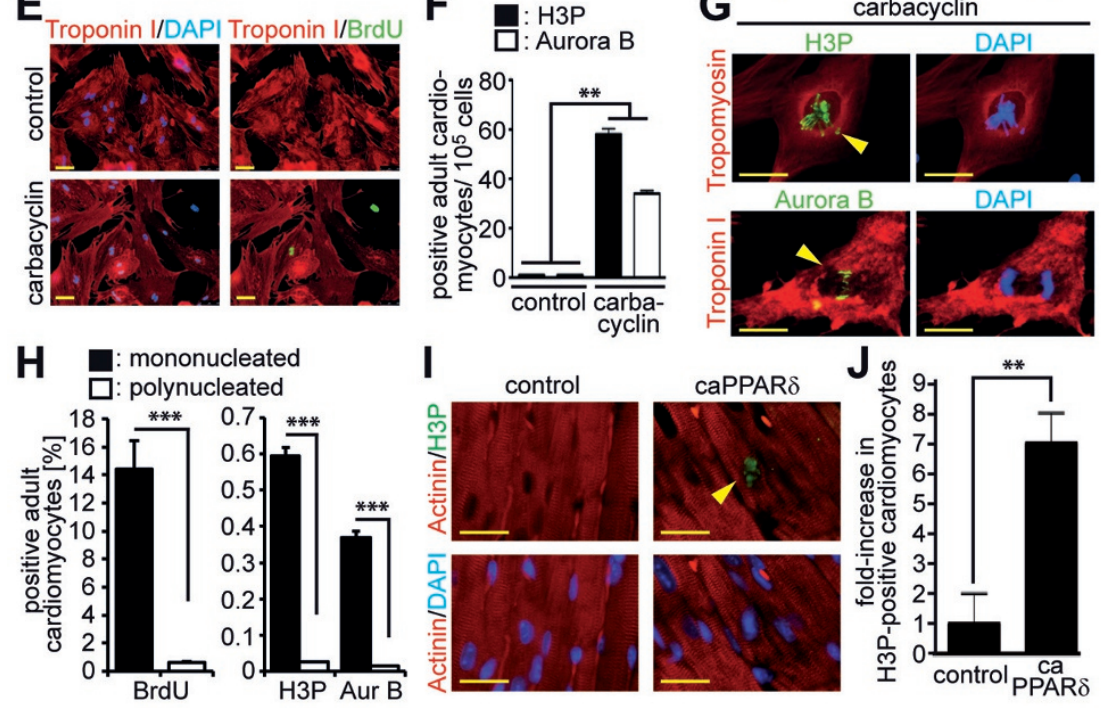

Figure 5 PPAR $\delta$ activity is sufficient for adult cardiomyocyte cell cycle re-entry. (A-C) Carbacyclin induces cell cycle re-entry of P8 cardiomyocytes. Quantitative analysis of $\mathrm{BrdU}$ incorporation $(\mathbf{A}, \mathbf{B}, n=4)$ and mitosis based on $\mathrm{H} 3 \mathrm{P}$ staining $(\mathbf{C}, n=$ 4) in mono- and binucleated cardiomyocytes. The overall percentage of binucleated cardiomyocytes in these cultures was $41.5 \% \pm 6.1 \%$. (D, E) Carbacyclin induces mAG expression after infection with Ad-mAG-hGem(1/110) as well as BrdU incorporation in adult cardiomyocytes. Quantitative analysis $(n=6)$. (F, G) Carbacyclin treatment induces mitosis and cytokinesis in adult cardiomyocytes. (F) Quantitative analysis $(n=6)$. (G) Representative examples of adult cardiomyocytes undergoing mitosis and cytokinesis stained for Tropomyosin or Troponin I (red, cardiomyocyte-specific) and H3P or Aurora B (green). Scale bar $=50 \mu \mathrm{m}$. $(\mathrm{H})$ Percentage of mono- and binucleated cardiomyocytes within the group of BrdU-positive cardiomyocytes $(n=4)$. (I) Representative heart sections from TMCM mice (control) and tamoxifen inducible transgenic mice with cardiomyocyte-restricted overexpression of VP16-PPAR $\delta$ (caPPAR $\delta$ ) 2 weeks after tamoxifen injection. Sections were stained with anti-Actinin (red, cardiomyocytes-specific) and anti-H3P (green, stains mitotic cells) antibodies. Nuclei were visualized with DAPI. Scale bar $=25 \mu \mathrm{m}$. (J) Quantitative analysis of I $(n=4,3$ sections per heart). $* * P<0.01, * * * P<0.001$. 
and $61.7 \% \pm 7.3 \%$ of the Aurora B-positive adult cardiomyocytes were mononucleated (Figure $5 \mathrm{H}$ ). Considering that only $5.7 \% \pm 2.2 \%$ adult cardiomyocytes were mononucleated in these cultures, carbacyclin induced DNA synthesis in $\sim 14 \%$, mitosis in $\sim 0.6 \%$ and cytokinesis in $\sim 0.4 \%$ of mononucleated cardiomyocytes compared to control levels of $\sim 0.6 \%, \sim 0.025 \%$ and $\sim 0.014 \%$ in polynucleated adult cardiomyocytes, respectively. Taken together, our data demonstrate that administration of carbacyclin in vitro promotes cell cycle progression through $\mathrm{S}$ phase, mitosis and cytokinesis in mononuclear adult rat cardiomyocytes and thus at a lower level than in neonatal cardiomyocytes (Figure 2A and 2G), but at a similar lev$\mathrm{el}$ as in $\mathrm{P} 8$ cardiomyocytes (Figure 5A-5C).

To determine whether PPAR $\delta$ activation could induce mitosis in adult cardiomyocytes in vivo, we utilized a transgenic mouse model with tamoxifen inducible, cardiomyocyte-restricted overexpression of constitutively active PPAR $\delta$ (caPPAR $\delta$; VP16-PPAR $\delta$ ) $[27,30]$. We refer to tamoxifen inducible $\alpha \mathrm{MyHC}$-Mer-Cre-Mer controls as TMCM mice and animals showing cardiomyocyte-restricted overexpression of VP16-PPAR $\delta$ as TMVPD mice. Such animals were injected with tamoxifen and 14 days later analyzed for H3P-positive mitotic cardiomyocytes 14 days. Cardiac-specific overexpression of constitutively active PPAR $\delta$ resulted in a 7-fold higher number of mitotic adult cardiomyocytes $(2.33 \pm 0.4$ vs $0.33 \pm 0.3$ per section, Figure 5I and 5J). These data suggest that activation of PPAR $\delta$ promotes cardiomyocyte proliferation in vitro and in vivo.

Activation of PPAR improves heart function after myocardial infarction

To determine whether PPAR $\delta$ activation is sufficient to improve the ineffective myocardial repair that takes place after myocardial infarction (MI) in mice, we induced MI by ligation of the left anterior descending artery (LAD) in adult TMCM (control) and TMVPD (caPPAR $\delta$ ) mice (Figure 6A). Analysis of trichrome-stained cross-sections of the heart two weeks post MI showed that the infarct size was reduced in mice overexpressing caPPAR $\delta$ (Figure $6 \mathrm{~B}$ and $6 \mathrm{C}$ ). Echocardiography also revealed that cardiomyocyte-restricted overexpression of caPPAR $\delta$ in adult TMVPD mice also improved heart function two weeks after MI. caPPAR $\delta$ mice showed shorter systolic endocardial and epicardial length compared to control TMCM mice, implicating less dilated systolic hearts in caPPAR $\delta$ expression compared to control animals (Supplementary information, Table S2). Consistently, the change in endocardial area measured in hearts expressing constitutively active PPAR $\delta$ was greater than that in control hearts (Supplementary information, Table S2). In addition, hearts with constitutively active PPAR $\delta$ showed an improved percentile fractional area change (FAC\%) compared to control hearts, indicating improved cardiac contraction (Figure 6D). Consequently, hearts expressing caPPAR $\delta$ showed increased endocardial stroke volume and cardiac output (Supplementary information, Table S2).

TMVPD animals with cardiac-specific overexpression of constitutively active PPAR $\delta$ showed a substantial increase in several parameters 2 weeks after MI relative to control TMCM mice. These included an increase in BrdU incorporation over 10 days in cardiomyocytes in the scar area ( $>9$-fold), the border zone $(>8$-fold) and in the remote zone (11-fold) compared to post-MI TMCM control mice (Scar area: $21.9 \% \pm 3.7 \%$ vs $2.41 \% \pm 0.2 \%$, infarct border zone: $10.92 \% \pm 1.7 \%$ vs $1.23 \% \pm 0.2 \%$, remote area: $1.76 \% \pm 0.2 \%$ vs $0.16 \% \pm 0.03 \%$, Figure $6 \mathrm{E}$ and $6 \mathrm{~F})$. Moreover, post-MI caPPAR $\delta$ mice showed a greater than 10-fold increase in mitotic (H3P-positive, $20.3 \pm 2$ vs $2 \pm 0.4$ per section) adult cardiomyocytes and a greater than 9-fold increase in cytokinetic (Aurora B-positive, $9 \pm 1.15$ vs $1 \pm 0.29$ per section) adult cardiomyocytes compared to post-MI control mice (Figure 6G$6 \mathrm{~J})$. Together, these data indicate that overexpression of PPAR $\delta$ following MI results in a marked beneficial effect by reducing infarct size, stimulating cardiomyocyte proliferation and improving cardiac function. This is further supported by the observation that Tbx20, whose overexpression has recently been demonstrated to promote adult cardiomyocyte proliferation and to improve cardiac function post MI [8], is significantly upregulated in caPPAR $\delta$ mice post MI (Figure 6K-6M). In contrast, no change in the number of c-Kit-positive cells was observed suggesting that the recruitment of endogenous heart progenitors plays either no role or a limited role during cardiac repair upon activation of PPAR $\delta$ (Supplementary information, Figure S7).

To determine whether PPAR $\delta$ regulates Tbx 20 expression at the transcriptional level, we generated a series of pGL3-Basic plasmid constructs for luciferase reporter assays, which contained Tbx20 promoter fragments with or without putative peroxisome proliferators response elements (PPRE) sites (identified by MatInspector software). When PPAR $\delta$ was co-transfected the reporter activity was significantly increased in constructs containing the $-1150 \mathrm{bp}$ region of the Tbx20 promoter with the PPRE element, suggesting that PPAR $\delta$ enhances the promoter activity of Tbx20. Co-transfection of PPAR $\gamma$ had no effect (Supplementary information, Figure S8).

Notably, the number of mitotic (H3P-positive) cardiomyocytes 2 weeks post $\mathrm{MI}$ in control mice was more than six-fold $(2.0 \pm 0.4$ per section $)$ higher than in un- 
A
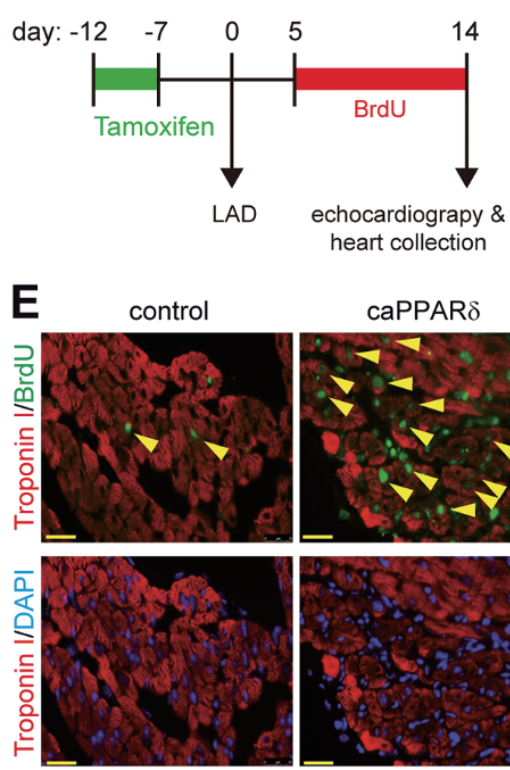

I

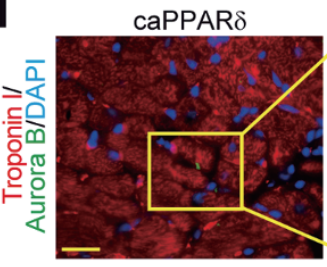

caPPAR $\delta$
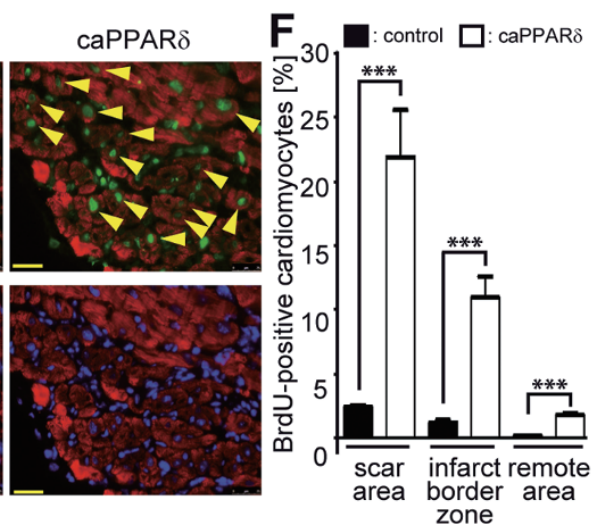

zone
B

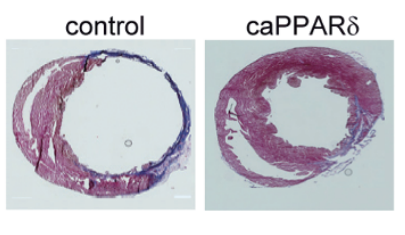

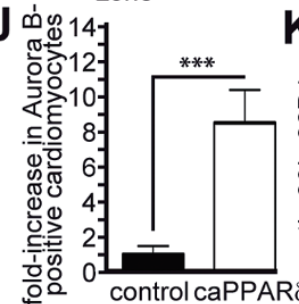

C

D

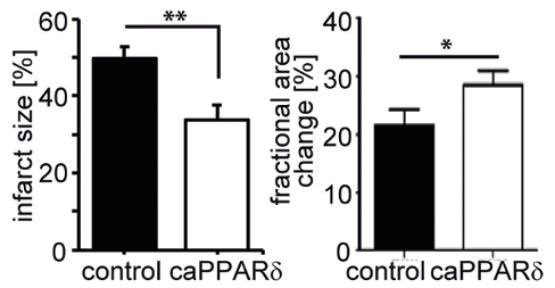

G

H
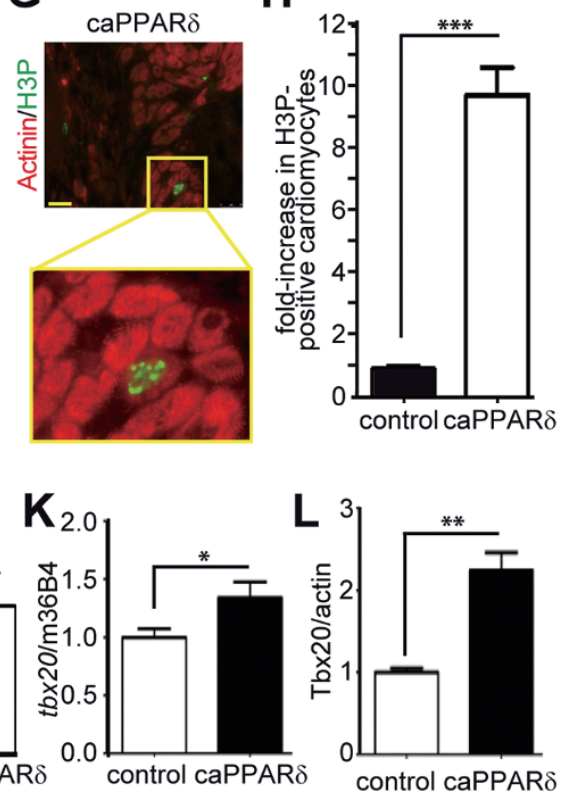

M

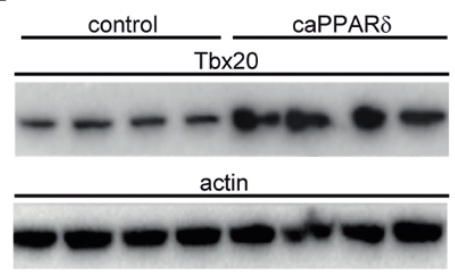

N
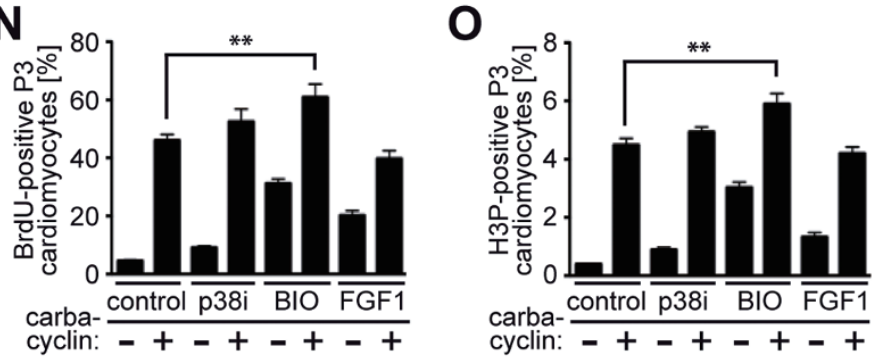

Figure 6 Activation of PPAR induces cardiomyocyte cell cycle re-entry in vivo and rescues cardiac function after myocardial infarction. Cardiomyocyte-restricted overexpression of a constitutively active PPAR $\delta$ was induced in adult mice utilizing TMVPD mice via tamoxifen injections (caPPAR $\delta$ ). One week after tamoxifen injection, MI was induced via LAD ligation and hearts were analyzed 2 weeks later. (A) Experimental design. (B) Representative images of hearts from caPPAR $\delta$ mice and control mice (TMCM) after Masson's Trichrome staining 2 weeks post MI. (C) Quantitative analysis of infarct size $(n=5)$ and (D) echocardiographic measurement demonstrating improved cardiac morphology and function post MI in caPPAR $\delta$ mice $(n$ $=7$ ) compared to control mice $(n=8)$. (E) Representative heart sections showing the scar area from control and caPPAR $\delta$ mice. Sections were stained with anti-Troponin I (red, cardiomyocytes-specific) and anti-BrdU (green, stains cycling cells) antibodies. Nuclei were visualized with DAPI. Arrowheads: indicate BrdU-positive cardiomyocytes. (F) Quantitative analysis of BrdU-positive cardiomyocytes ( $n=4,3$ sections per heart). (G) Representative mitotic cardiomyocyte (Actinin: red, cardiomyocytes-specific; H3P: green, stains mitotic cells) in a heart section from caPPAR $\delta$. (H) Quantitative analysis of H3P-positive cardiomyocytes ( $n=4,3$ sections per heart). (I) Representative heart section from caPPAR $\delta$ mice stained for Troponin I (red, cardiomyocytes-specific) and Aurora B (green, stains cells in cytokinesis). Nuclei were visualized with DAPI. Arrow: indicates a dividing cardiomyocyte. Asterix: indicates a dividing non-myocyte. (J) Quantitative analysis of Aurora B-positive cardiomyocytes ( $n=4$, 3 sections per heart). (K) Quantitative analysis of tbx20 mRNA via real-time PCR. m36B4 was used as control $(n=7)$. (L, M) Tbx20 protein expression was assessed via western blot analysis $(n=4) . * P<0.05, * * * P<0.001$. Scale bar $=$ $25 \mu \mathrm{m}$. (N, O) Quantitative analysis of an additive effect on carbacyclin-induced BrdU incorporation by p38 MAP kinase inhibitor SB203580, fibroblast growth factor 1 (FGF1) or GSK3 $\beta$ inhibitor BIO. ${ }^{* *} P<0.01, n=6$. 


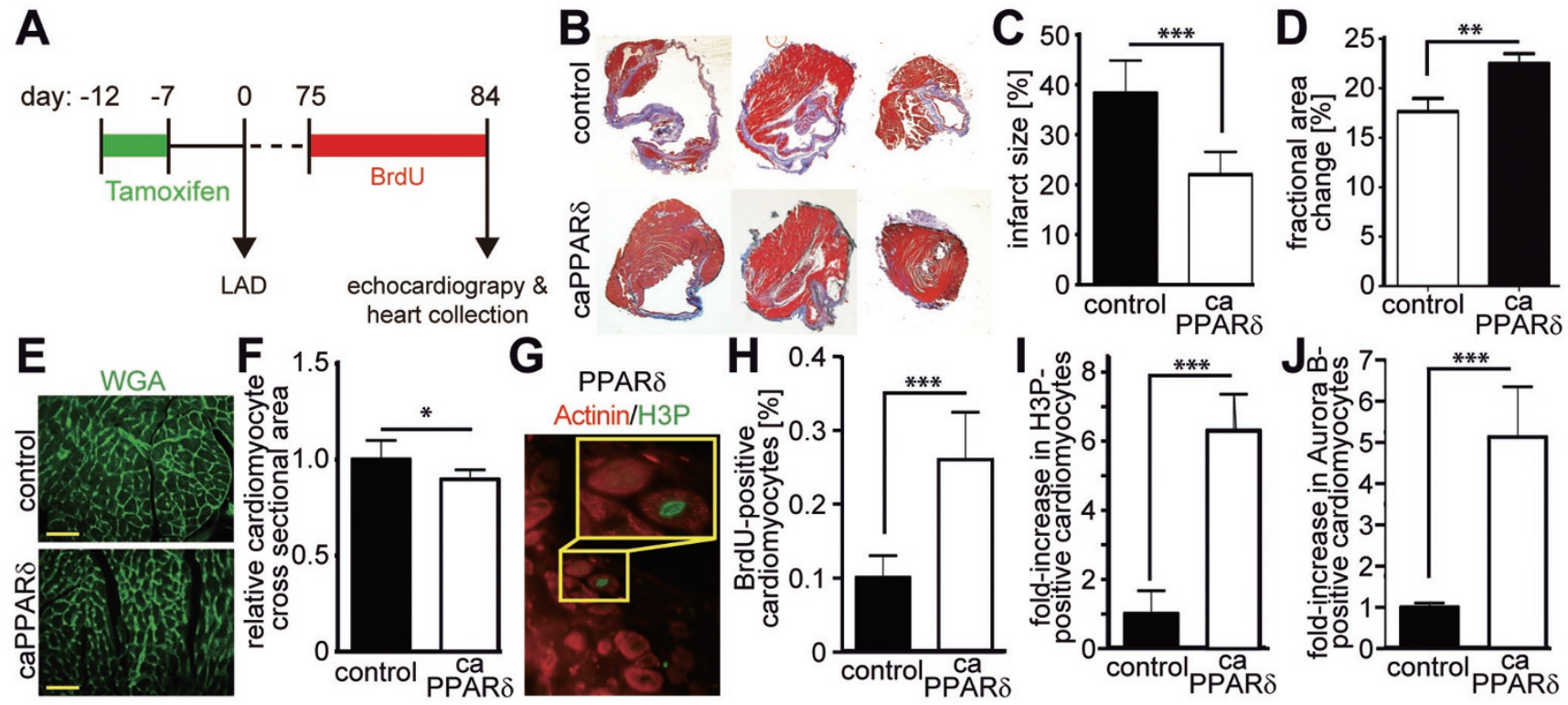

Figure 7 Activation of PPAR $\delta$ induces cardiomyocyte cell cycle re-entry in vivo and rescues cardiac function after myocardial infarction (MI). Cardiomyocyte-restricted overexpression of a constitutively active PPAR $\delta$ was induced in adult mice utilizing TMVPD mice via tamoxifen injections (caPPAR $\delta$ ). One week after tamoxifen injection, LAD ligation was performed and hearts were analyzed 84 days later. (A) Experimental design. (B) Representative images of hearts from caPPAR $\delta$ mice and control mice (TMCM) (three different levels of the same heart) after Masson's Trichrome staining at 84 days post MI. (C) Quantitative analysis of infarct size (caPPAR $\delta: n=5$, control: $n=3$ ) and (D) echocardiographic measurement demonstrating improved cardiac function post MI in caPPAR $\delta$ mice $(n=9)$ compared to control mice $(n=8)$. (E) Representative heart sections from control and caPPAR $\delta$ mice. Sections were stained with wheat germ agglutinin (WGA, green, cell membranes) to determine cardiomyocyte cross sectional area. (F) Quantitative analysis of $\mathbf{E}(n=4)$. (G) Representative heart sections from control and caPPAR $\delta$ mice stained for Actinin (red, cardiomyocytes-specific) and H3P (green, stains mitotic cells). (H-J) Quantitative analysis of BrdU-, H3P- and Aurora B-positive cardiomyocytes $(n=4,3$ sections per heart). $* P<0.05, * * P<0.01, * * * P<0.001$. Scale bar $=25 \mu \mathrm{m}$.

injured control hearts $(0.33 \pm 0.3$ per section $)$ and more than eight-fold ( $20.3 \pm 2$ per section) higher in caPPAR $\delta$ mice than in uninjured caPPAR $\delta$ mice $(2.33 \pm 0.4$ per section) (Figures 5 and 6). These data indicate that the MI itself induces a signaling pathway, which promotes the effect of caPPAR $\delta$. Previously, it has, e.g., been shown that cardiac injury causes activation of Wnt $/ \beta$-catenin signaling [31] that in turn induces cardiomyocyte proliferation in vitro [32] and improves heart function in vivo [33]. Notably, it is also known that PPAR $\delta$ regulates glycogen synthase kinase 3 beta $(\mathrm{GSK} 3 \beta) / \beta$-catenin signaling $[34,35]$. We therefore assessed whether carbacyclin activates the GSK $3 \beta / \beta$-catenin-pathway in cardiomyocytes in vitro. We found that carbacyclin stimulation resulted in phosphorylation of GSK3 $\beta$ at Ser9 (Supplementary information, Figure S9A), an increase in nuclear PPAR $\delta$ and $\beta$-catenin (Supplementary information, Figure S9B and S9C), and increased TCF reporter activity (Supplementary information, Figure S9D). Furthermore, overexpression of DN $\beta$-catenin ( $\beta$-cat) or DN-TCF, and addition of $15 \mu \mathrm{M}$ FH535, an inhibitor of nuclear
$\operatorname{PPAR} \delta / \beta$-catenin complexes, substantially reduced carbacyclin-induced BrdU incorporation (Supplementary information, Figure S9E). Taken together, these data indicate that PPAR $\delta$ promotes cardiomyocyte proliferation via GSK3 $\beta / \beta$-catenin signaling and that induction of Wnt/ $\beta$-catenin signaling in the injured heart promotes cardiomyocyte cell cycle entry in caPPAR $\delta$ mice after MI. This hypothesis is supported by the fact that the addition of $5 \mu \mathrm{M}$ BIO, a GSK3 inhibitor, but not FGF1 or p38i, markedly increased the number of carbacyclin-induced BrdU-positive and H3P-positive cardiomyocytes in culture (Figure $6 \mathrm{~N}$ and $6 \mathrm{O}$ ).

\section{PPARS activation post MI has long-term beneficial ef-} fects

To determine whether overexpression of caPPAR $\delta$ following MI has a long-term beneficial effect, we performed a separate double-blinded study inducing MI by LAD ligations in control adult TMCM mice and in mice expressing constitutively active PPAR $\delta$ (Figure 7A). Analysis of trichrome-stained heart cross sections 84 

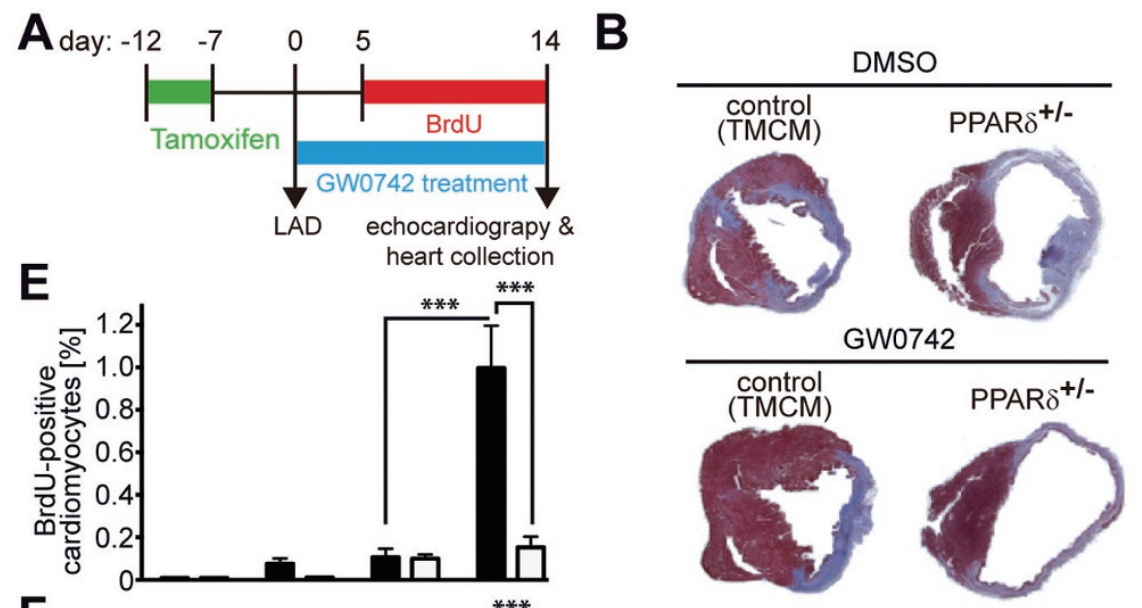

GW0742
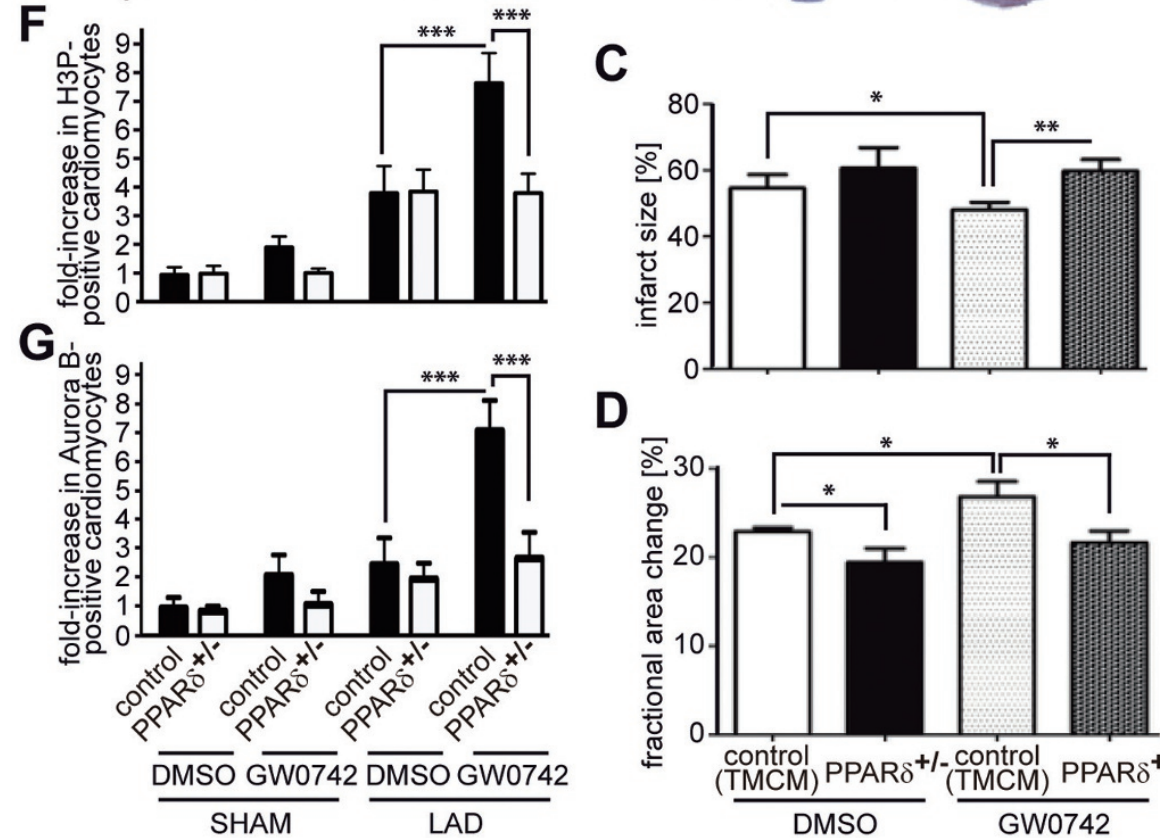

D

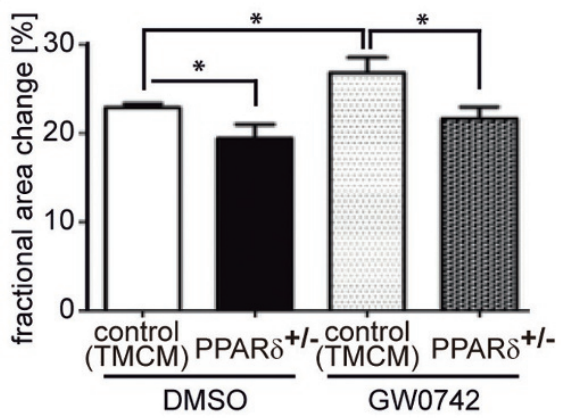

Figure 8 GW0742 treatment induces cardiomyocyte cell cycle progression and rescues heart function and myocardial infarction (MI). (A) Experimental design. One week after tamoxifen injections, LAD ligation was performed. Hearts were analyzed after 2 weeks of GW0742 treatment. (B) Representative images of PPAR $\delta^{+/}$mice and control mice (TMCM) treated with DMSO or GW0742 after Masson's Trichrome staining 2 weeks post MI. (C) Quantitative analysis of infarct size $(n=5)$ and (D) echocardiographic measurement demonstrating improved cardiac function post MI in PPAR $\delta^{+/-}$mice (DMSO: $n=8$; GW0742: $n=12$ ) compared to control mice (DMSO: $n=9$; GW0742: $n=8$ ). (E-G) Quantitative analysis of BrdU-, H3P- and Aurora B-positive cardiomyocytes ( $n=4,3$ sections per heart). $* P<0.05, * * P<0.01,{ }^{*} * * P<0.001$.

days post MI showed that the infarct size was reduced by almost $40 \%$ in the caPPAR $\delta$ mice (Figure $7 \mathrm{~B}$ and $7 \mathrm{C}$ ). Echocardiography revealed that cardiomyocyte-restricted overexpression of caPPAR $\delta$ in adult TMVPD mice exerted protective effects on the heart 84 days after MI (Supplementary information, Table S3) resulting in improved FAC $\%$ in caPPAR $\delta$ hearts compared to control hearts (Figure 7D). Of note, as we have shown previously [27], overexpression of caPPAR $\delta$ did not result in cardiomyocyte hypertrophy but rather in its inhibition, based on the cardiomyocyte cross sectional area determined by wheat germ agglutinin (WGA) staining (Figure 7E and $7 \mathrm{~F}$ ). Thus, the greater amount of muscle area compared to control hearts (reduced scar area, Figure 7B and 7C) is due to an increased number of cardiomyocytes and not hypertrophy.

At 84 days after MI and upon 10 days of BrdU treatment, the number of BrdU-positive $(0.26 \pm 0.038$ vs. 0.1 $\pm 0.017, P<0.01)$, mitotic (H3P-positive) $(3 \pm 0.29$ vs. $0.5 \pm 0.15$ per section, $P<0.01$ ), and cytokinetic (Aurora 
B-positive) ( $1.47 \pm 0.2$ vs. $0.25 \pm 0.02$ per section, $P<$ $0.01)$ cardiomyocytes was markedly higher in caPPAR $\delta$ mice compared to control mice (Figure 7G-7J). This indicates active cardiomyocyte proliferation. However, the proliferation indices 84 days after MI was substantially lower than those at 2 weeks post MI suggesting that factors/signaling pathways induced by the MI, such as GSK $3 \beta / \beta$-catenin signaling, transiently enhanced the effect of PPAR $\delta$ activation. Together, these data demonstrate that activation of PPAR $\delta$ after MI exerts a longterm beneficial effect on heart function.

\section{Drug-based activation of PPAR $\delta$ preserves heart function after myocardial infarction}

To determine whether a therapeutic approach toward activating PPAR $\delta$ induces cardiomyocyte cell cycle progression exerting beneficial effects on the post-MI heart, we performed a third double-blinded study (Figure 8A) utilizing the well-characterized and highly specific PPAR $\delta$ activator GW0742. Analysis of trichrome-stained heart cross sections 2 weeks post MI showed that the infarct size was substantially reduced in TMCM control mice treated with GW0742 (Figure 8B and 8C). Echocardiography revealed that GW0742 treatment (Supplementary information, Table S4) resulted in improved FAC $\%$ in GW0742- compared to DMSO-treated animals 14 days post MI (Figure 8D).

GW0742 treatment markedly increased the number of BrdU-positive $(0.93 \% \pm 0.17 \%$ vs $0.1 \% \pm 0.04 \%, P$ $<0.01$ ), mitotic (H3P positive) $4 \pm 0.32$ vs $2 \pm 0.29$ per section, $P<0.01)$ and cytokinetic (Aurora B-positive) $(2.0$ \pm 0.17 vs $0.7 \pm 0.15$ per section, $P<0.01)$ cardiomyocytes compared to DMSO-treated animals 2 weeks after MI (Figure 8E-8G). Notably, improvement in scarring, cardiac function and proliferation markers upon GW0742 treatment was abolished in $\mathrm{TMPD}^{+/-}$mice harboring a heterozygous, cardiac-restricted deletion of PPAR $\delta$ $\left(\mathrm{PPAR}^{+/-}\right.$; Figure 8B-8G, note: homozygous deletion of PPAR $\delta$ impairs heart function [25]). These data support the notion that PPAR $\delta$ in cardiomyocytes is required for the effect of PPAR $\delta$ ligand-induced cardiomyocyte proliferation.

Together, these data suggest that small molecule-based activation of PPAR $\delta$ after MI induces cardiomyocyte cell cycle progression and exerts a beneficial effect by reducing infarct size and improving cardiac function.

\section{Discussion}

In this study, we modified the Fucci system [12] by integrating a cardiomyocyte-specific promoter to establish a fluorescence-based live imaging-screening assay to identify new inducers of $\mathrm{P} 3$ rat cardiomyocyte proliferation. This system eliminates the need for laborious and expensive techniques such as immunofluorescence staining, incorporation of nucleotide analogs or cell count assays. Most importantly, we identified carbacyclin as a novel potential inducer of P3 cardiomyocyte proliferation. Subsequent proliferation assays have demonstrated that carbacyclin induces proliferation of P3, P8 and preferentially mononuclear adult rat cardiomyocytes via PPAR $\delta$. Conversely, inhibition of PPAR $\delta$ inhibited cardiomyocyte proliferation during zebrafish heart regeneration. Notably, PPAR $\delta$ activation following MI has both short-term and long-term beneficial effects improving scarring, cardiac function and expression of proliferation markers. Our results confirm the hypothesis that conserved signaling pathways in P3 rat cardiomyocytes are required for cardiomyocyte proliferation during cardiac regeneration in zebrafish and have the potential to promote proliferation of mononuclear adult mammalian cardiomyocyte and cardiac repair.

Our data indicate that PPAR $\delta$ activation mainly acts on a mononucleated subpopulation of cardiomyocytes that maintains a fetal/neonatal phenotype, as the pro-proliferative effect on cardiomyocytes in vitro was markedly decreased with the aging of the stimulated cardiomyocytes. This decline in efficiency with age has previously been reported for several factors resulting in the "subpopulation theory", which was recently supported by a hypoxia fate-mapping study that identified cycling cardiomyocytes in the adult heart $[3,10]$.

Previously, it has been shown that PPAR $\delta$ is expressed in human heart [36]. In addition, activation of PPAR $\delta$ signaling has been demonstrated to modulate proliferation of other human cell types such as epithelial cells [37-39], endothelial progenitor cells [40], keratinocytes [15] and liposarcoma cell lines [41]. In addition, we have shown here that activation of PPAR $\delta$ signaling promotes proliferation of hiPSC-derived cardiomyocytes. Moreover, PPAR $\delta$ has the same role in mice and humans $[42$, 43]. These data indicate that activation of PPAR $\delta$ signaling after MI might be of relevance in the human clinical setting.

While our finding that PPAR $\delta$ regulates cardiomyocyte proliferation is novel, it is known that PPAR $\delta$ overexpression has a positive effect on heart function after cardiac injury. Yet there is no report on a positive effect after MI. For example, myocardial injury due to I/R injury, as determined by measuring the infarcted area relative to the area at risk, was significantly reduced by cardiac-specific PPAR $\delta$ overexpression in adult heart concomitant with increased myocardial glucose utilization [26]. In a subsequent study, Liu and coworkers showed 
that PPAR $\delta$ overexpression in adult heart elevates oxidative metabolism concomitant with an increased mitochondrial DNA copy number and an enhanced cardiac performance. While transverse aortic constriction in PPAR $\delta$ transgenic mice resulted in the same hypertrophic response as in control mice, dilation of the ventricles as well as fibrosis was significantly reduced [27]. Yet, none of these studies has demonstrated that the metabolic changes accounted for the observed beneficial effects. Interestingly, glucose utilization has previously been associated with proliferation and regeneration. For example, proliferative fetal cardiomyocytes, in contrast to postmitotic postnatal cardiomyocytes, utilize mainly glucose [44] and Lin28a-mediated increase in glycolysis and oxidative phosphorylation enhances tissue repair in several adult tissues [45]. Thus, it will be interesting in future studies to investigate whether alterations of energy metabolism can promote cardiomyocyte proliferation.

That PPAR $\delta$ activation has a great potential for cardiac repair and is supported by the fact that PPAR $\delta$ has been associated with wound healing and tissue repair in other organs and tissues [16-19]. Our observation that PPAR $\delta$ activation has a stronger effect after MI than in uninjured hearts resulted in the hypothesis that the effect of PPAR $\delta$ activation is enhanced by GSK $3 \beta / \beta$-catenin signaling. Previously, it has been shown that $\mathrm{Wnt} / \beta$-catenin signaling plays important roles during vertebrate heart development and is re-activated in response to cardiac injury $[31,46]$. Moreover, inhibition of GSK3 $\beta$ promotes cardiomyocyte proliferation in vitro [32] and is in vivo cardioprotective, and induces physiological hypertrophy as well as cardiomyocyte proliferation [33, 47]. However, in contrast to PPAR $\delta$ activation, GSK-3 $\beta$ inhibition leads to excessive fibrosis after MI through hyperactivation of profibrotic TGF- $\beta 1-S M A D-3$ signaling [33, 48-50]. This indicates that PPAR $\delta$ activation and GSK3 $\beta$ inhibition do not share the same downstream signaling pathways. Therefore, it needs to be tested if the pro-regenerative effect of PPAR $\delta$ activation can be enhanced by GSK3 $\beta$ inhibitors in vivo, as we have observed in vitro, and whether this treatment is effective if applied after injury or during chronic heart failure. In addition, one has to consider that PPAR $\delta$ activation has also been associated with the development of tumors in animal studies [24, $51]$ and proliferative diseases $[14,15]$. Thus, in order to make use of the PPAR $\delta$ and the PPAR $\delta / \beta$-catenin synergy in a therapeutic setting, it might be necessary in the future to develop tools for cardiomyocyte-specific activation of PPAR $\delta$ and $\beta$-catenin or for the local delivery of their agonists or modified RNAs at optimized concentrations.

Our data demonstrate that activation of PPAR $\delta$ sig- naling is the key for cardiomyocyte proliferation. Even though we initially identified the PPAR $\delta$ agonist carbacyclin in our screen, we utilized GW0742 to demonstrate the importance of PPAR $\delta$ signaling in in vivo studies, as GW0742 is a well-characterized and highly specific PPAR $\delta$ activator [52]. In contrast, carbacyclin is a PPAR $\delta$ activator with prostacyclin effects [53] that might have obscured the interpretation of the in vivo results. However, our in vitro data indicate that carbacyclin is more potent than GW0742 in inducing cardiomyocyte proliferation. Carbacyclin might be a more potent PPAR $\delta$ activator than GW0742, but might also activate additional pro-proliferative pathways. Therefore, future studies are warranted to further determine if non-PPAR $\delta$ actions of carbacyclin may synergistically optimize its cardiomyocyte proliferation effect in vivo.

Previously, we have shown that cardiomyocyte proliferation can be induced by activating $\beta$-catenin [32] or PI3 kinase signaling [28]. Here we show that the PI3 kinase pathway is not involved in cardiomyocyte proliferation induced by activation of PPAR $\delta$ signaling. This appears surprising as it has been shown that activation of PPAR $\delta$ stimulates hepatic stellate cell proliferation through the PI3 kinase/protein kinase-C alpha-/beta-mixed lineage kinase-3 pathway [54]. One possible explanation might be that activation of this pathway increases the phosphorylation of p38 MAPK and that inhibition of p38 MAPK abolished activated PPAR $\delta$-dependent stimulation of hepatic stellate cell proliferation. In contrast, we have shown that inhibition of p38 MAPK in cardiomyocytes promotes cardiomyocyte proliferation [28].

Heart diseases, which are often associated with cardiomyocyte loss, represent a significant socio-economic burden. Currently, there is no therapy available to efficiently treat heart disease. Therefore, the development of a cardiomyocyte proliferation screening system and the finding that activation of PPAR $\delta$ has the potential to improve cardiac function after MI, which could possibly be reinforced with GSK3 $\beta$ inhibitors, is potentially of great therapeutic value.

\section{Materials and Methods}

\section{Cardiomyocytes isolation, culture and stimulation}

The investigation conforms with the Guide for the Care and Use of Laboratory Animals published by the US National Institutes of Health (NIH Publication No. 85-23, revised 1996). The local Committee approved animal experiments for Care and Use of Laboratory Animals (Regierungspräsidium Darmstadt, Gen. Nr. B 2/Anz. 75). Ventricular cardiomyocytes from 3-day-old (P3), 8-day-old (P8) and 12-week-old (adult) Sprague-Dawley rats were isolated and cultured as described $[55,56]$. Cells were initially cultured for $48-72 \mathrm{~h}$ in the presence of $20 \mu \mathrm{M}$ cytosine-D-arab- 
inofuranoside and $5 \%$ horse serum before stimulation to prevent non-myocyte proliferation. Cardiomyocytes were subsequently treated in the presence of serum $(0.2 \%$ FCS: neonatal, $5 \%$ FCS: adult) with DMSO as a negative control, $1 \mu \mathrm{M}$ Carbacyclin if not stated otherwise (Enzo Life Science) or 100 nM GW0742 (Cayman Chemical). Postnatal cardiomyocytes were stimulated once; adult cardiomyocytes daily while the medium was exchanged every 3 days. As positive control $50 \mu \mathrm{g} / \mathrm{ml} \mathrm{FGF} 1+5 \mu \mathrm{M}$ SB203580 (p38i) were used. Signaling pathway inhibitors were added $1 \mathrm{~h}$ before stimulation: $20 \mu \mathrm{M}$ PHT-427 (Selleckchem), 100 nM GSK3787 (Santa Cruz Biotechnology), $15 \mu \mathrm{M}$ FH535 (Tocris Bioscience), $10 \mu$ M LY294002 (Sigma), 20 M PD98059 (Cell Signaling).

\section{Generation and stimulation of hiPSC-derived cardiomyo- cytes}

Human fibroblasts (GM05171, Coriell) were reprogrammed to iPSCs using the Stemgent mRNA Reprogramming Kit (00-0071), the Stemgent microRNA Booster Kit (00-0073) and the Stemgent Stemfect RNA Transfection Kit (00-0069). About $5.0 \times 10^{4}$ fibroblasts were plated onto Matrigel-coated six-well tissue culture plates and transfected with the microRNA cocktail on day 0 of reprogramming. On days 1-3, fibroblasts were transfected with the mRNA reprogramming cocktail daily, which consisted of Oct4, Sox2, Klf4, c-Myc, Lin28 and nGFP mRNAs in a 3:1:1:1:1:1 stoichiometric ratio. Fibroblasts were co-transfected with both the mRNA and microRNA reprogramming cocktails on day 4 , and then subsequently only transfected with the mRNA reprogramming cocktail daily from days 5-11. HiPSC colonies were manually selected from the fibroblast cultures after 2-3 days and cultured on Matrigel-coated 12-well plates. hiPSCs were cultured in E8 media and were split every 4 days. Differentiation along the cardiac lineage was induced as previously described [57]. Briefly, hiPSCs were maintained in E8 media and passaged every 4 days onto Matrigel-coated plates. On day 0 (start of differentiation) hiPSCs were treated with $1 \mathrm{mg} / \mathrm{ml}$ Collagenase B (Roche) for $1 \mathrm{~h}$, or until cells dissociated from plates, to generate embryoid bodies (EBs). Cells were collected and centrifuged at 1200 r.p.m. for $3 \mathrm{~min}$, and resuspended as small clusters of 50-100 cells by gentle pipetting in differentiation media (RPMI (Gibco), $2 \mathrm{mM}$ L-glutamine (Invitrogen), $4 \times 10^{4}$ monothioglycerol (MTG, Sigma), $50 \mu \mathrm{g} / \mathrm{ml}$ ascorbic acid (Sigma)). Differentiation media was supplemented with $2 \mathrm{ng} / \mathrm{ml} \mathrm{BMP4}$ and $3 \mu \mathrm{mol}$ Thiazovivin (Milipore) on day 0 . EBs were maintained in $6 \mathrm{~cm}$ dishes at 37 ${ }^{\circ} \mathrm{C}$ in $5 \% \mathrm{CO}_{2}, 5 \% \mathrm{O}_{2}$ and $90 \% \mathrm{~N}_{2}$. On day 1 , media was changed to differentiation media supplemented with $20 \mathrm{ng} / \mathrm{ml} \mathrm{BMP} 4,20 \mathrm{ng} / \mathrm{ml}$ Activin A, $5 \mathrm{ng} / \mathrm{ml} \mathrm{bFGF}$ (all R\&D Systems) and $1 \mu \mathrm{mol}$ Thiazovivin (Milipore). On day 3, EBs were harvested and washed once with DMEM (Gibco). Media was changed to differentiation media supplemented with $5 \mathrm{ng} / \mathrm{ml} \mathrm{VEGF} \mathrm{(R \& D} \mathrm{Systems)} \mathrm{and} 5 \mu \mathrm{mol} / 1$ XAV (Stemgent). On day 5, media was changed to differentiation media supplemented with $5 \mathrm{ng} / \mathrm{ml}$ VEGF (R\&D Systems). After day 8 , media was changed to differentiation media without supplements and was renewed every 3-4 days. hiPSC-derived cardiomyocytes were then stimulated at day 23 with carbacyclin.

\section{Screening}

P3 rat cardiomyocytes were seeded in $100 \mu 1$ medium at a density of 15000 cells per 96 well for 2 days. Then cells were infected with Ad-mAG-hGem(1/110). After $24 \mathrm{~h}$ they were washed and treated with compounds dissolved in DMSO, and $0.2 \%$ FCS $(\mathrm{Nu}-$ clear Receptor Ligand Library, 74 compounds, Enzo Life Science; Epigenetics Screening Library, 54 compounds, Cayman Chemicals). AG expression was analyzed every $12 \mathrm{~h}$ (for quantitative analysis a random field of around 100 cells was evaluated) for the following 4 days by visual inspection using a Leica fluorescence microscope. The maximal number of mAG-hGem(1/110)-positive cells was used to normalize the data against the DMSO-treated control as a fold change. Hit compounds were defined as those giving an effect greater than 2-fold.

\section{Adenovirus infection}

mCherry-hCdt1(30/120) and mAG-hGem(1/110) were PCR amplified from lentiviral vectors [12] and cloned into pAlpha-MyHC (clone 26) using SalI [58] to generate adenoviruses (Sirion Biotech $\mathrm{GmbH}$ ). Cardiomyocytes were infected 1 (adult) or 2 (P3) days after isolation at a multiplicity of infection (MOI.) of 400. Infection efficiency of cardiomyocytes was $>90 \%$. For inhibition experiments, cultures were infected with adenoviruses expressing DN-Akt (200 MOI) [59], DN- $\beta$-catenin and DN-TCF4 (both 100 MOI, Vector Biolabs) 2 days after isolation. Cells were washed after $24 \mathrm{~h}$ and treated.

\section{Immunofluorescence staining}

Staining was performed as described $[28,55,56]$. Primary antibodies: mouse monoclonal anti-mCherry (1:200, Clontech, 632543 or 1:50, DSHB, 3A11), anti-Tropomyosin (1:200, Sigma, T9283), anti-sarcomeric alpha Actinin (1:100, Abcam, ab9465), anti-Aurora B (1:200, BD Transduction Laboratories, 611083), anti-p27 (1:50, BD Transduction Laboratories, 610242), anti-Ki67 (1:250, Abcam, ab8191), rabbit polyclonal anti-Troponin I (sc-15368), anti-Cyclin A (sc-751), anti-cdc2 (sc-954), anti-Geminin (sc-13015) (all 1:50, Santa Cruz), anti-phospho-Histone H3 (Ser10) (1:200, Millipore, 06-570), anti-pRb807/811 (1:100, Cell Signaling, 9308), anti-mAG (1:300, MBL, PM011), anti p27 (1:50, SantaCruz, sc528), anti-survivin (1:50, Novus, NB500-201K8), rat monoclonal anti-BrdU (1:100, Abcam, ab6326) and goat polyclonal anti c-kit (1:100, R\&D Systems, AF1356). Immune complexes were detected with ALEXA 488- or ALEXA 594-conjugated secondary antibodies (1:200; Molecular Probes). DNA was visualized with DAPI (4', 6'-diamidino-2-phenylindole, $0.5 \mathrm{~g} / \mathrm{ml}$ ). For BrdU, cells were cultured in $30 \mu \mathrm{M}$ BrdU (neonatal: last $24 \mathrm{~h}$, adult: last 5 days).

\section{RT-PCR analysis}

Total RNA was isolated using the RNeasy Kit (Qiagen). RT reaction was performed using the oligo(dT) primer (Qiagen). PCR was performed according to standard protocols. Primers: p21: forward 5'-AGGCAGACCAGCCTAACAGA-3', reverse, 5'-CAGCACTA AGGAGCCTACCG-3', c-myc: forward (F) 5'-CGAGCTGAAGCGTAGCTTTT-3', reverse (R) 5'-CTCGCCGTTTCCTCAGTAAG-3', $\beta$-catenin: F 5'-ACAGCACCTTCAGCACTCT-3', R 5'-AAGTTCTTGGCTATTACGAC-3', PPAR $\delta$ : F 5'-GAACAGCCACAGGAGGAGAC-3', R 5'-CCCATCACAGCCCATCTG-3', cyclin B: F 5'-GCGTAAAGTCAGCGAACAGTCAAG-3', R 5'-gcGGAGAGGGAGTATCAACCAAA-3', cyclin A: F 5'-GCGTATTTGCCATCGCTTATTGCT-3', R 5'-GCGCTGTGGTGCTTTGAGGTAGGT-3', cyclin D2: F 5'-AAGAGAGAGGCGTGTTCGTC-3', R 5'-TTCCTTCTTGGGTTCAATGC-3', gapdh: F 5'-CAGAAGACTGTGGATGGCCC-3', R 5'-AGTGTAGCCCAGGATGCCCT-3'. 


\section{Western blotting}

Protein extracts were prepared and western blot analyses performed as described [60]. Briefly, equal amount of proteins was resolved by $10 \%$ Novex Bis-Tris Gels (Invitrogen) and blotted onto nitrocellulose membranes. Membranes were blocked (5\% non-fat dry milk (DM) or 5\% BSA in Tris-buffered saline (TBS: $10 \mathrm{mM}$ Tris- $\mathrm{HCl}$ (pH 7.5), $150 \mathrm{mM} \mathrm{NaCl}$ ) with $0.1 \%$ Tween, $1 \mathrm{~h}, \mathrm{RT}$ ) and incubated with primary antibodies diluted in $5 \% \mathrm{milk} / \mathrm{TBS} / \mathrm{T}$ and/ or $5 \% \mathrm{BSA} / \mathrm{TBS} / \mathrm{T}$ (overnight, $4{ }^{\circ} \mathrm{C}$ ): rabbit polyclonal anti-Cyclin D2 (1:1 000, sc-593), anti-PPAR $\delta$ (1:500, sc-7197) (both Santa Cruz, DM), anti-phospho-GSK-3 (DM, 9336), anti-pan-actin (DM, 4968), anti-PDK1 (BSA, 3062) (all 1:1 000, Cell Signaling), rabbit monoclonal anti- $\beta$-catenin (1:1 000, BSA, 9582), anti-GSK-3 $\beta$ (1:1 000, DM, 9315), mouse monoclonal phospho (308) anti-Akt (1:1 000, BSA, 9275), anti-Akt (1:1 000, DM, 2920) (all Cell Signaling), anti-KIP/p27 (1:2 500, DM, 610241), anti-PARP (1:1 000, DM, 611038) (all BD Transduction Laboratories). Antigen/antibody complexes were visualized using horseradish peroxidase-conjugated secondary antibodies (Amersham) and Super Signal@ECL detection system (Bio-Rad).

\section{RNA interference}

For siRNA knockdown, cardiomyocytes were transfected 48 to $72 \mathrm{~h}$ after seeding by lipofectamine RNAiMAX kit (Invitrogen) with validated siRNAs or All Stars Negative Control siRNA (Qiagen) $(100 \mathrm{nM})$, washed after $4 \mathrm{~h}$, and stimulated after $48 \mathrm{~h}$. The efficiency of siRNAs was verified by RT-PCR.

\section{Luciferase assay}

TOPflash or FOPflash reporter plasmids ( $2 \mu \mathrm{g}$, Upstate Signaling) were co-transfected with $2 \mu \mathrm{g}$ pGL4.75 (hRluc/CMV) vector (Promega) into 2 Mio cells (Nucleofactor Kit, Amaxa, efficiency of transfection: $30 \%$ [61]). Cells were cultured 3 days in 24-well plates and subsequently stimulated with compounds for $24 \mathrm{~h}$. Reporter activity was measured by using the Dual Luciferase Assay System (Promega). TOPflash or FOPflash activity was normalized to Renilla luciferase activity of pGL4.75, an internal standard for transfection efficiency.

\section{Tbx20 promoter analysis}

A series of Tbx20 promoter reporter constructs and plasmids expressing either PPAR $\delta$ or PPAR $\gamma$ were co-transfected into HEK293 cells using Lipofectamine 2000 (Invitrogen). Forty-eight hours after transfection, the cells were lysed with positive lysis buffer (Promega) and the firefly and renilla luciferase activities in the lysate were determined using a luminometer. The Tbx20 promoter luciferase activities were normalized to renilla luciferase activities.

\section{Zebrafish cryoinjury and PPAR inhibitor (GSK3787) treat- ment}

All procedures involving zebrafish were approved by local animal experiments committees and performed in compliance with animal welfare laws, guidelines and policies, according to national and European law. Wild-type Gol or $m y l 7: \mathrm{GFP}^{\mathrm{twu} 34 \mathrm{Tg}}$ zebrafish [62] aged $\sim 8$ months were used. Heart cryoinjury was performed as described previously [63] except that a liquid nitrogen cooled copper filament was used instead of dry ice to induce cryoinjury. After the injury, fish were maintained under standard housing conditions till
6 days post injury (dpi). They were then kept in aquarium water containing either DMSO solvent or PPAR $\delta$ inhibitor (GSK3787; 5 $\mu \mathrm{M})$ for a duration of $24 \mathrm{~h}$. At $7 \mathrm{dpi}$, hearts were harvested, fixed and cryosectioned as described previously [63].

\section{Zebrafish immunofluorescence and cell quantification}

Primary antibodies used were anti-PCNA (1:2 000; Dako \#M0879), anti-mef2 (1:50; Santa Cruz Biotechnology \#sc-313) and anti-H3P (1:1 000, Cell Signaling Technology \#9706). DAPI was used to stain nuclei. Images shown are single optical planes acquired using a Leica SP5 confocal microscope. For quantification of PCNA positive cardiomyocytes, the percentage of Mef2-positive cells also positive for PCNA was quantified within a zone that extended $150 \mu \mathrm{m}$ from the wound border. For each heart 2-3 sections displaying the biggest wounds were analyzed. H3P-positive cardiomyocytes were quantified on all heart sections, which contained an injury area (average eight sections per heart). In this case the total cardiomyocyte number in the border zone was estimated by determining the average density of cardiomyocytes per $\mu \mathrm{m}^{2}$ in three separate areas (size: $75 \mu^{2}$ ) and by multiplying this number by the total border area size.

\section{PPAR $\delta$ mouse model, myocardial infarction, echocardiog-} raphy and BrdU treatment

All experimental procedures were conducted in accordance with the Guide for Care and Use of Laboratory Animals, and were approved by the Institutional Animal Care and Use Committee of the University of Alabama at Birmingham (UAB). The utilized transgenic mouse line TMVPD line allows tamoxifen inducible cardiomyocyte-restricted overexpression of a constitutively active mutant PPAR $\delta$ gene (VP16-PPAR $\delta$ ) and has been described previously $[27,30,64]$. This line was generated by crossing a transgenic line expressing VP16-PPAR $\delta$ under the control of the human cytomegalovirus immediate early enhance/chicken $\beta$-actin promoter with tamoxifen inducible $\alpha$ MyHC-Mer-Cre-Mer (TMCM) transgenic mice. $\mathrm{TMPD}^{+/}$mice harbor a heterozygous, cardiac-restricted deletion of PPAR $\delta$, which were generated by crossing floxed PPAR $\delta$ mice with TMCM mice. Tamoxifen injection once daily for 5 days induced cardiomyocyte-restricted PPAR $\delta$ overexpression (1.8-fold [27]). MI was induced 1 week after tamoxifen administration by ligation of the left anterior descending coronary artery (LAD). Coronary artery ligation was performed on 3 months old, male mice. Briefly, mice were anesthetized with isoflurane and a catheter was inserted into the trachea. The chest was opened through a left parasternal incision to expose the heart at the left 3rd-4th intercostal space. The pericardium was opened, and ligations were made on the left anterior descending coronary artery using 8-0 silk sutures. The lungs were slightly overinflated to assist in removal of air in the pleural cavity. For the drug-based approach, GW0742 treatment started on the same day as the surgery by subcutaneous injection at a dose of $1 \mathrm{mg} / \mathrm{kg}$ until sacrifice, using 10\% DMSO/Saline as vehicle. The in vivo effect of GW0742 on activating PPAR $\delta$ target genes has previously been reported [65]. For BrdU (Sigma) labeling, BrdU was dissolved in drinking water at a final concentration of $1 \mathrm{mg} / \mathrm{ml}$ and provided to the mice daily for 10 days before harvesting tissue for paraffin sections. BrdU-containing water bottles were shielded from light to prevent BrdU degradation and the water was replaced twice per week. A high-resolution echocardiograph system (VisualSonic VEVO 770 
System) was used to assess cardiac structure/function in vivo with a $35 \mathrm{MHz}$ probe before terminal experiments. All data and images were saved and analyzed by an Advanced Cardiovascular Package Software (VisualSonic VEVO 770 System) under EKV mode.

\section{Immunostaining and histology of heart sections}

Mice were anesthetized and hearts were perfused (cardioplegic buffer), fixed (4\% paraformaldehyde), embedded (paraffin) and sectioned ( $5 \mu \mathrm{m}$ thick). Following antigen retrieval in boiling EDTA Buffer (1 M EDTA, pH 8.0, 8 min), sections were cooled to room temperature (RT), blocked ( $5 \%$ goat serum $/ 0.2 \%$ Tween- 20 / PBS, $30 \mathrm{~min}$ ), incubated overnight at $4{ }^{\circ} \mathrm{C}$ with rabbit anti-phospho-Histone H3 (Ser10) (1:200, Millipore, 06-570) and mouse anti-sarcomeric alpha Actinin (1:100, Abcam, ab9465) or mouse anti-Aurora B (1:200, BD Transduction Laboratories, \#611083) and rabbit anti-Troponin I (1:50, Santa Cruz, sc-15368) washed with PBS, and incubated with corresponding secondary antibodies conjugated to Alexa Fluor 488 and Alexa Fluor 594 (Invitrogen). Nuclei were visualized with DAPI. Hematoxylin/eosin and Masson's Trichrome staining were used to assess cardiac morphology and fibrosis. Infarct size was calculated according to the formula: (length of coronal infarct perimeter (epicardial + endocardial)/ total left ventricle coronal perimeter (epicardial + endocardial) $) \times$ 100 [66]. For the serial section analysis in Figure 5, the software MIQaunt was utilized [67].

\section{Wheat germ agglutinin staining}

To determine cardiomyocyte cross sectional areas, slides were rinsed three times after deparaffinization in PBS and then incubated for $1 \mathrm{~h}$ at RT with WGA conjugated to Alexa Fluor 488 (50 $\mu \mathrm{g} / \mathrm{ml}$, Invitrogen, CA, USA). Slides were then rinsed in PBS and mounted in Vectashield (Vector Labs, CA, USA). To quantify the size of cells, images at $20 \times$ magnification were analyzed using ImageJ. Quantitative analyses involved counting of multiple fields from four independent hearts per group, and three sections per heart ( $\sim 50$ cells per field assessed, total $\sim 250$ cells per sample).

\section{Statistical analysis}

For immunofluorescence analyses, 50 cardiomyocytes in five random fields of two different subpopulations were counted per experiment (total: 500 cardiomyocytes) based on previous experiences. Data of at least three independent experiments are expressed as mean \pm SEM. For animal studies, group sizes (at least six) were estimated according to previous experience and power calculation. Before the experiment and data analysis, animals were randomized to new cages by a scientist who was not involved in this project. Thus, the investigator who performed surgery and echocardiographic assessment was not able to recognize group information during the experiment and data analysis. Animals were excluded from the study based on pre-established criteria: (1) genotyping errors; (2) transgenic induction errors and (3) surgical errors. Results were analyzed by Graph Pad Prism (version 4.00, Graph Pad Software). During data analysis, the investigator was blinded to the group allocation. All group information was revealed after data analysis was finalized. Statistical significance was determined using a two-tailed Student's $t$-test and analysis of variance. The values of $P<0.05$ were considered statistically significant.

\section{Acknowledgments}

We thank Ingrid Hauck-Schmalenberger and Jana Petzold for technical assistance, Atsushi Miyawaki for Fucci constructs, Jeffrey Robbins for the MHC construct and Kenneth Walsh for the DN-Akt adenovirus, and Mauro Giacca for critical reading of the manuscript. This work was supported by the Alexander von Humboldt Foundation (Sofja Kovalevskaja Award, FBE), the IRTG 1566 (PROMISE, DFG, FBE), the Emerging Fields Initiative Cell "Cycle in Disease and Regeneration (CYDER)" (Friedrich-Alexander-University Erlangen-Nürnberg, FBE), by the German Research Foundation (DFG, INST 410/91-1 FUGG to FBE), the Collaborative Research Center 1149 (DFG, GW), a "Klaus-Georg-und-Sigrid-Hengstberger-Forschungsstipendium" (DGK, GW), a Basic Science Award (\#7-12-BS-208, American Diabetes Association, QY), a Grant-in-aid from American Heart Association (AHA, QY), a Pre-doctoral Fellowship (AHA, YD), and the Small Animal Phenotyping Core of the UAB Diabetes Research Center (DRC) supported by the National Institute of Diabetes and Digestive and Kidney Diseases (NIDDK, P30DK079626).

\section{Author Contributions}

AM performed, assisted by HVR, all in vitro experiments and all histological analyses except the analysis of scar area. AM, YD, LH, TK and KY performed all in vivo experiments and the analysis regarding scaring and Tbx20 expression. QL performed the Tbx20 promoter analysis. MDV performed all zebrafish experiments. NW and ND generated hiPSC-derived cardiomyocytes. FBE, GW, QY and AM designed the experiments and wrote the manuscript. All authors were involved in the analysis and interpretation of the data.

\section{Competing Financial Interests}

The authors declare no competing financial interests.

\section{References}

1 Mozaffarian D, Benjamin EJ, Go AS, et al. Heart disease and stroke statistics-2016 update: a report from the American Heart Association. Circulation 2015; 133:e599.

2 Kikuchi K, Poss KD. Cardiac regenerative capacity and mechanisms. Annu Rev Cell Dev Biol 2012; 28:719-741.

3 Leone M, Magadum A, Engel FB. Cardiomyocyte proliferation in cardiac development and regeneration: a guide to methodologies and interpretations. Am J Physiol Heart Circ Physiol 2015; 309:H1237-1250.

4 Sdek P, Zhao P, Wang Y, et al. Rb and p130 control cell cycle gene silencing to maintain the postmitotic phenotype in cardiac myocytes. J Cell Biol 2011; 194:407-423.

5 Puente BN, Kimura W, Muralidhar SA, et al. The oxygen-rich postnatal environment induces cardiomyocyte cell-cycle arrest through DNA damage response. Cell 2014; 157:565-579.

6 Zebrowski DC, Vergarajauregui S, Wu CC, et al. Developmental alterations in centrosome integrity contribute to the post-mitotic state of mammalian cardiomyocytes. eLife 2015; 4:e05563.

7 Zebrowski DC, Becker R, Engel FB. Towards regenerating the mammalian heart: challenges in evaluating experimentally 
induced adult mammalian cardiomyocyte proliferation. Am J Physiol Heart Circ Physiol 2016; 310:H1045-1054.

8 Xiang FL, Guo M, Yutzey KE. Overexpression of Tbx20 in adult cardiomyocytes promotes proliferation and improves cardiac function after myocardial infarction. Circulation 2016; 133:1081-1092.

9 Tao G, Kahr PC, Morikawa Y, et al. Pitx2 promotes heart repair by activating the antioxidant response after cardiac injury. Nature 2016; 534:119-123.

10 Kimura W, Xiao F, Canseco DC, et al. Hypoxia fate mapping identifies cycling cardiomyocytes in the adult heart. Nature 2015; 523:226-230.

11 Bersell K, Arab S, Haring B, Kuhn B. Neuregulin1/ErbB4 signaling induces cardiomyocyte proliferation and repair of heart injury. Cell 2009; 138:257-270.

12 Sakaue-Sawano A, Kurokawa H, Morimura T, et al. Visualizing spatiotemporal dynamics of multicellular cell-cycle progression. Cell 2008; 132:487-498.

13 Tan NS, Vazquez-Carrera M, Montagner A, Sng MK, Guillou H, Wahli W. Transcriptional control of physiological and pathological processes by the nuclear receptor PPARbeta/ delta. Prog Lipid Res 2016; 64:98-122.

14 Bishop-Bailey D. PPARs and angiogenesis. Biochem Soc Trans 2011; 39:1601-1605.

15 Schug TT, Berry DC, Shaw NS, Travis SN, Noy N. Opposing effects of retinoic acid on cell growth result from alternate activation of two different nuclear receptors. Cell 2007; 129:723-733.

16 Anderson SP, Yoon L, Richard EB, Dunn CS, Cattley RC, Corton JC. Delayed liver regeneration in peroxisome proliferator-activated receptor-alpha-null mice. Hepatology 2002; 36:544-554

17 Angione AR, Jiang C, Pan D, Wang YX, Kuang S. PPARdelta regulates satellite cell proliferation and skeletal muscle regeneration. Skelet Muscle 2011; 1:33.

18 Michalik L, Desvergne B, Tan NS, et al. Impaired skin wound healing in peroxisome proliferator-activated receptor (PPAR) alpha and PPARbeta mutant mice. J Cell Biol 2001; 154:799814.

19 Nakamura Y, Nakamura T, Tarui T, Inoue J, Kinoshita S. Functional role of PPARdelta in corneal epithelial wound healing. Am J Pathol 2012; 180:583-598.

20 Kliewer SA, Forman BM, Blumberg B, et al. Differential expression and activation of a family of murine peroxisome proliferator-activated receptors. Proc Natl Acad Sci USA 1994; 91:7355-7359.

21 Evans RM, Barish GD, Wang YX. PPARs and the complex journey to obesity. Nat Med 2004; 10:355-361.

22 Barak Y, Liao D, He W, et al. Effects of peroxisome proliferator-activated receptor delta on placentation, adiposity, and colorectal cancer. Proc Natl Acad Sci USA 2002; 99:303-308.

23 Peters JM, Lee SS, Li W, et al. Growth, adipose, brain, and skin alterations resulting from targeted disruption of the mouse peroxisome proliferator-activated receptor beta(delta). Mol Cell Biol 2000; 20:5119-5128.

24 Barlaka E, Galatou E, Mellidis K, Ravingerova T, Lazou A. Role of pleiotropic properties of peroxisome proliferator-activated receptors in the heart: focus on the nonmetabolic effects in cardiac protection. Cardiovasc Ther 2016; 34:37-48.
25 Cheng L, Ding G, Qin Q, et al. Cardiomyocyte-restricted peroxisome proliferator-activated receptor-delta deletion perturbs myocardial fatty acid oxidation and leads to cardiomyopathy. Nat Med 2004; 10:1245-1250.

26 Burkart EM, Sambandam N, Han X, et al. Nuclear receptors PPARbeta/delta and PPARalpha direct distinct metabolic regulatory programs in the mouse heart. J Clin Invest 2007; 117:3930-3939.

27 Liu J, Wang P, Luo J, et al. Peroxisome proliferator-activated receptor $\{$ beta $\} /\{$ delta $\}$ activation in adult hearts facilitates mitochondrial function and cardiac performance under pressure-overload condition. Hypertension 2011; 57:223-230.

28 Engel FB, Schebesta M, Duong MT, et al. p38 MAP kinase inhibition enables proliferation of adult mammalian cardiomyocytes. Genes Dev 2005; 19:1175-1187.

29 Pollock CB, Yin Y, Yuan H, et al. PPARdelta activation acts cooperatively with 3-phosphoinositide-dependent protein kinase-1 to enhance mammary tumorigenesis. PLoS One 2011; 6:e16215.

30 Kim T, Zhelyabovska O, Liu J, Yang Q. Generation of an inducible, cardiomyocyte-specific transgenic mouse model with PPAR beta/delta overexpression. Methods Mol Biol 2013; 952:57-65.

31 Ozhan G, Weidinger G. Wnt/beta-catenin signaling in heart regeneration. Cell Regen 2015; 4:3.

32 Tseng AS, Engel FB, Keating MT. The GSK-3 inhibitor BIO promotes proliferation in mammalian cardiomyocytes. Chem Biol 2006; 13:957-963.

33 Lal H, Ahmad F, Woodgett J, Force T. The GSK-3 family as therapeutic target for myocardial diseases. Circ Res 2015; 116:138-149.

34 Jeong AY, Lee MY, Lee SH, Park JH, Han HJ. PPARdelta agonist-mediated ROS stimulates mouse embryonic stem cell proliferation through cooperation of p38 MAPK and Wnt/beta-catenin. Cell Cycle 2009; 8:611-619.

35 Scholtysek C, Katzenbeisser J, Fu H, et al. PPARbeta/delta governs Wnt signaling and bone turnover. Nat Med 2013; 19:608-613.

36 Lee WS, Kim J. Peroxisome proliferator-activated receptors and the heart: lessons from the past and future directions. PPAR Res 2015; 2015:271983.

37 Montagner A, Wahli W, Tan NS. Nuclear receptor peroxisome proliferator activated receptor (PPAR) beta/delta in skin wound healing and cancer. Eur J Dermatol 2015; 25 Suppl 1:4-11.

38 Jeong E, Koo JE, Yeon SH, Kwak MK, Hwang DH, Lee JY. PPARdelta deficiency disrupts hypoxia-mediated tumorigenic potential of colon cancer cells. Mol Carcinog 2014; 53:926937.

39 Nagy TA, Wroblewski LE, Wang D, et al. Beta-catenin and p120 mediate PPARdelta-dependent proliferation induced by Helicobacter pylori in human and rodent epithelia. Gastroenterology 2011; 141:553-564.

40 He T, Smith LA, Lu T, Joyner MJ, Katusic ZS. Activation of peroxisome proliferator-activated receptor- $\{$ delta $\}$ enhances regenerative capacity of human endothelial progenitor cells by stimulating biosynthesis of tetrahydrobiopterin. Hypertension 2011; 58:287-294.

41 Wagner KD, Benchetrit M, Bianchini L, Michiels JF, Wag- 
ner N. Peroxisome proliferator-activated receptor beta/delta (PPARbeta/delta) is highly expressed in liposarcoma and promotes migration and proliferation. J Pathol 2011; 224:575588.

42 Alvarez-Guardia D, Palomer X, Coll T, et al. PPARbeta/delta activation blocks lipid-induced inflammatory pathways in mouse heart and human cardiac cells. Biochim Biophys Acta 2011; 1811:59-67.

43 Luz-Crawford P, Ipseiz N, Espinosa-Carrasco G, et al. PPARbeta/delta directs the therapeutic potential of mesenchymal stem cells in arthritis. Ann Rheum Dis 2016; 75:2166-2174.

44 Taegtmeyer H, Sen S, Vela D. Return to the fetal gene program: a suggested metabolic link to gene expression in the heart. Ann NY Acad Sci 2010; 1188:191-198.

45 Shyh-Chang N, Zhu H, Yvanka de Soysa T, et al. Lin28 enhances tissue repair by reprogramming cellular metabolism. Cell 2013; 155:778-792.

46 Heallen T, Zhang M, Wang J, et al. Hippo pathway inhibits Wnt signaling to restrain cardiomyocyte proliferation and heart size. Science 2011; 332:458-461.

47 Woulfe KC, Gao E, Lal H, et al. Glycogen synthase kinase-3beta regulates post-myocardial infarction remodeling and stress-induced cardiomyocyte proliferation in vivo. Circ Res 2010; 106:1635-1645.

48 Iwaisako K, Haimerl M, Paik YH, et al. Protection from liver fibrosis by a peroxisome proliferator-activated receptor delta agonist. Proc Natl Acad Sci USA 2012; 109:E1369-E1376.

$49 \mathrm{Su} \mathrm{X}$, Zhou G, Wang Y, et al. The PPARbeta/delta agonist GW501516 attenuates peritonitis in peritoneal fibrosis via inhibition of TAK1-NFkappaB pathway in rats. Inflammation 2014; 37:729-737.

50 Teunissen BE, Smeets PJ, Willemsen PH, De Windt LJ, Van der Vusse GJ, Van Bilsen M. Activation of PPARdelta inhibits cardiac fibroblast proliferation and the transdifferentiation into myofibroblasts. Cardiovasc Res 2007; 75:519-529.

51 Sahebkar A, Chew GT, Watts GF. New peroxisome proliferator-activated receptor agonists: potential treatments for atherogenic dyslipidemia and non-alcoholic fatty liver disease. Expert Opin Pharmacother 2014; 15:493-503.

52 Sznaidman ML, Haffner CD, Maloney PR, et al. Novel selective small molecule agonists for peroxisome proliferator-activated receptor delta (PPARdelta) - synthesis and biological activity. Bioorg Med Chem Lett 2003; 13:1517-1521.

53 Whittle BJ, Steel G, Boughton-Smith NK. Gastrointestinal actions of carbacyclin, a stable mimic of prostacyclin. J Pharm Pharmacol 1980; 32:603-604.

54 Kostadinova R, Montagner A, Gouranton E, et al. GW501516-activated PPARbeta/delta promotes liver fibrosis via $\mathrm{p} 38$-JNK MAPK-induced hepatic stellate cell proliferation. Cell Biosci 2012; 2:34.

55 Engel FB, Hauck L, Boehm M, Nabel EG, Dietz R, von Harsdorf R. p21(CIP1) Controls proliferating cell nuclear antigen level in adult cardiomyocytes. Mol Cell Biol 2003; 23:555-565.

56 Engel FB, Hauck L, Cardoso MC, Leonhardt H, Dietz R, von

Harsdorf R. A mammalian myocardial cell-free system to study cell cycle reentry in terminally differentiated cardiomyocytes. Circ Res 1999; 85:294-301.

57 Yang L, Soonpaa MH, Adler ED, et al. Human cardiovascular progenitor cells develop from a KDR+ embryonic-stem-cell-derived population. Nature 2008; 453:524-528.

58 Gulick J, Subramaniam A, Neumann J, Robbins J. Isolation and characterization of the mouse cardiac myosin heavy chain genes. J Biol Chem 1991; 266:9180-9185.

59 Fujio Y, Walsh K. Akt mediates cytoprotection of endothelial cells by vascular endothelial growth factor in an anchorage-dependent manner. J Biol Chem 1999; 274:16349-16354.

60 Novoyatleva T, Diehl F, van Amerongen MJ, et al. TWEAK is a positive regulator of cardiomyocyte proliferation. Cardiovasc Res 2010; 85:681-690.

61 Gresch O, Engel FB, Nesic D, et al. New non-viral method for gene transfer into primary cells. Methods 2004; 33:151163.

62 Huang CJ, Tu CT, Hsiao CD, Hsieh FJ, Tsai HJ. Germ-line transmission of a myocardium-specific GFP transgene reveals critical regulatory elements in the cardiac myosin light chain 2 promoter of zebrafish. Dev Dyn 2003; 228:30-40.

63 Schnabel K, Wu CC, Kurth T, Weidinger G. Regeneration of cryoinjury induced necrotic heart lesions in zebrafish is associated with epicardial activation and cardiomyocyte proliferation. PLoS One 2011; 6:e18503.

64 Wang P, Liu J, Li Y, et al. Peroxisome proliferator-activated receptor delta is an essential transcriptional regulator for mitochondrial protection and biogenesis in adult heart. Circ Res 2010; 106:911-919.

65 Wagner N, Jehl-Pietri C, Lopez P, et al. Peroxisome proliferator-activated receptor beta stimulation induces rapid cardiac growth and angiogenesis via direct activation of calcineurin. Cardiovasc Res 2009; 83:61-71.

66 Bergmann O, Bhardwaj RD, Bernard S, et al. Evidence for cardiomyocyte renewal in humans. Science 2009; 324:98-102.

67 Nascimento DS, Valente M, Esteves T, et al. MIQuant semi-automation of infarct size assessment in models of cardiac ischemic injury. PLoS One 2011; 6:e25045.

(Supplementary information is linked to the online version of the paper on the Cell Research website.)

(c) (i)

This work is licensed under a Creative Commons Attribution 4.0 Unported License. The images or other third party material in this article are included in the article's Creative Commons license, unless indicated otherwise in the credit line; if the material is not included under the Creative Commons license, users will need to obtain permission from the license holder to reproduce the material. To view a copy of this license, visit http:// creativecommons.org/licenses/by/4.0/

C The Author(s) 2017 